\title{
BOUNDED HOLOMORPHIC FUNCTIONS ON FINITE RIEMANN SURFACES
}

\author{
BY \\ E. L. STOUT(1)
}

1. Introduction. This paper is devoted to the study of some problems concerning bounded holomorphic functions on finite Riemann surfaces. Our work has its origin in a pair of theorems due to Lennart Carleson.

The first of the theorems of Carleson we shall be concerned with is the following [7]:

THEOREM 1.1. Let $f_{1}, \cdots, f_{n}$ be bounded holomorphic functions on $U$, the open unit disc, such that $\left|f_{1}(z)\right|+\cdots+\left|f_{n}(z)\right| \geqq \delta>0$ holds for some $\delta$ and all $z$ in $U$. Then there exist bounded holomorphic function $g_{1}, \cdots, g_{n}$ on $U$ such that $f_{1} g_{1}+\cdots+f_{n} g_{n}=1$.

In $\S 2$, we use this theorem to establish an analogous result in the setting of finite open Riemann surfaces. $\S \S 3$ and 4 consider certain questions which arise naturally in the course of the proof of this generalization. We mention that the chief result of $\S 2$, Theorem 2.6 , has been obtained independently by N. L. Alling [3] who has used methods more highly algebraic than ours.

The second matter we shall be concerned with is that of interpolation. If $R$ is a Riemann surface and if $E$ is a subset of $R$, call $E$ an interpolation set for $R$ if for every bounded complex-valued function $\alpha$ on $E$, there is a bounded holomorphic function $f$ on $R$ such that $f \mid E=\alpha$. Carleson [6] has characterized interpolation sets in the unit disc:

THEOREM 1.2. The set $\left\{z_{k}\right\}_{k=1}^{\infty}$ of points in $U$ is an interpolation set for $U$ if and only if there exists $\delta>0$ such that for all $n$

$$
\delta \leqq \prod_{k=1 ; k \neq n}^{\infty}\left|\frac{z_{k}-z_{n}}{1-\bar{z}_{n} z_{k}}\right| .
$$

An alternative proof is to be found in $[12$, p. 203].

In $\S \S 5,6$ and 7 we obtain several characterizations of interpolation sets

Presented to the Society, August 27, 1964 under the title Interpolation on finite Riemann surfaces; received by the editors June 17, 1964.

(1) The research for this paper was supported by National Science Foundation Grant GP 2235. 
in finite open Riemann surfaces. Our theorems in these sections will depend essentially on the results of $\S 2$.

Heretofore we have not made precise the class of Riemann surfaces on which we shall be working. We shall always assume given a Riemann surface $R$ which is embedded in a compact Riemann surface $R_{0}$ in such a way that $\partial R$, the boundary of $R$ in $R_{0}$ consists of finitely many analytic simple closed curves $\Gamma_{1}, \cdots, \Gamma_{n}$. We also assume that $R_{0} \backslash R=\Delta_{1} \cup \cdots \cup \Delta_{m}$ where each $\Delta_{j}$ is homeomorphic to the closed unit disc. We shall refer to $R$ as a finite Riemann surface.

Inasmuch as most of the results of the first seven sections are invariant under analytic homeomorphisms, the following question naturally arises: Given a Riemann surface $S$, what conditions guarantee the existence of a compact Riemann surface $R_{0}$ and an analytic homeomorphism $\phi$ of $S$ into $R_{0}$ such that if $R=\phi[S]$, then $R$ and $R_{0}$ stand in the relation indicated in the last paragraph? $\S 8$ is devoted to this question. We obtain a criterion for the existence of such a surface $R_{0}$ and such a map $\phi$ for a given surface $S$. The criterion is in terms of the topological properties of $S$ and the algebraic properties of the algebra of bounded analytic functions on $S$.

Certain notations will be used consistently in the course of our work. We will always use $U$ to denote the open unit disc: $U=\{z:|z|<1\}$. The boundary of $U$, the unit circle, will be denoted by $\Lambda$. If $R$ is a Riemann surface, we will denote by $H_{\infty}[R]$ the space of all bounded functions which are holomorphic on $R$. If $f \in H_{\infty}[R]$, then $\|f\|_{R}$ will be the number $\sup \{|f(z)|: z \in R\}$. The function $\|\cdot\|_{R}$ is a norm on $H_{\infty}[R]$, and under this norm, $H_{\infty}[R]$ is a Banach algebra. This latter fact will play a fundamental role in certain of our considerations. We shall use $\mathfrak{M}[R]$ to denote the space of nonzero complex homomorphisms of $H_{\infty}[R]$ taken together with the weak* topology. Occasionally, when there is no danger of confusion, $\mathfrak{M}[R]$ will be abbreviated to $\mathfrak{M}$.

This paper represents the major portion of the author's doctoral dissertation. The author takes this opportunity to express his gratitude to Professor Walter Rudin for his continued interest and encouragement during the preparation of this dissertation. The author also acknowledges a very useful conversation with Dr. Michael Voichick concerning the possibility of a theorem like Theorem 7.1.

A preliminary form of some of our results appeared in [17], and a more extensive summary of our results, but no proofs, appeared in [18].

2. The corona problem. In this section we shall establish a generalization of Theorem 1.1 in the setting of a finite open Riemann surface, and we shall establish certain complementary results.

We begin by considering the case of a domain $D$ in the plane which is doubly connected. We assume that the complement of $D$ with respect to the closed plane consists of two nondegenerate components. By means of the uniformization theorem, we can regard $H_{\infty}[D]$ as the closed subalgebra of $H_{\infty}[U]$ consisting 
of those $f \in H_{\infty}[U]$ which are invariant under a certain group of conformal maps of $U$ onto itself. This group, which we shall denote by $G$, is in an infinite cyclic group.

THEOREM 2.1. Let $f_{1}, \cdots, f_{n}$ be elements of $H_{\infty}[U]$ which are invariant under $G$ and which satisfy $\left|f_{1}(z)\right|+\cdots+\left|f_{n}(z)\right| \geqq \delta$ for some $\delta>0$ and all $z$ in $U$. Let there exist $g_{1}, \cdots, g_{n}$ in $H_{\infty}[U]$ such that $\sum_{j=1}^{n} f_{j} g_{j}=1$ and such that $\max \left\|g_{j}\right\|_{U} \leqq M$. Then there exist $\tilde{g}_{1}, \cdots, \tilde{g}_{n}$ in $H_{\infty}[U]$ which are invariant under $G$, which satisfy $\sum_{j=1}^{n} f_{j} \tilde{g}_{j}=1$, and which also satisfy $\max \left\|\tilde{g}_{j}\right\|_{U} \leqq M$.

Proof. Define $\mathscr{H}$ to be the direct sum of $n$ copies of $H_{\infty}[U]$. In $\mathscr{H}$, let $S$ be the set of all $n$-tuples $\left\langle h_{1}, \cdots, h_{n}\right\rangle$ such that $\sum_{j=1}^{n} f_{j} h_{j}=1$ and $\max _{j}\left\|h_{j}\right\|_{U} \leqq M$. Our hypotheses are exactly that $S$ is nonvoid. It is clear that $S$ is a convex subset of $\mathscr{H}$.

Let $\tau$ be the topology of coordinatewise almost uniform convergence in $\mathscr{H}$ so that $\left\langle h_{1}^{(m)}, \cdots, h_{n}^{(m)}\right\rangle \stackrel{\tau}{\rightarrow}\left\langle h_{1}, \cdots, h_{n}\right\rangle$ if and only if $h_{j}^{(m)}$ converges to $h_{j}$ uniformly on compact subsets of $U$ for $j=1, \cdots, n$. The topology $\tau$ is metrizable, and a normal families argument shows that $S$ is $\tau$-compact.

We define a group of operators $\left\{\Phi_{\gamma}\right\}_{\gamma \in G}$ on $\mathscr{H}$ by means of

$$
\Phi_{\gamma}\left\langle h_{1}, \cdots, h_{n}\right\rangle=\left\langle h_{1} \circ \gamma, \cdots, h_{n} \circ \gamma\right\rangle
$$

for all $\gamma \in G$. The operators $\Phi_{\gamma}$ are all $\tau$-continuous, and they all take the compact, convex set $S$ onto itself. Since they commute, the fixed point theorem of Markov and Kakutani $\left[9\right.$, p. 456] affirms the existence of a point $\left\langle\tilde{g}_{1}, \cdots, \tilde{g}_{n}\right\rangle$ in $S$ which is invariant under the group $\left\{\Phi_{\gamma}\right\}_{\gamma \in G}$.

We see that the functions $\tilde{g}_{1}, \cdots, \tilde{g}_{n}$ have the desired property. q.e.d.

It is of interest to note that the theorem supplies a solution to the problem for the case of annular domains which is of smallest conceivable norm: With $f_{j}$ as in the theorem and $S$ as in the proof, let $A=\inf \left\{t\right.$ : there exist $g_{1}, \cdots, g_{m}$ in $H_{\infty}[U]$ such that $\sum f_{j} g_{j}=1$ and $\left.\max _{j}\left\|g_{j}\right\|_{U} \leqq t\right\}$. Let $S_{A}=\left\{\left\langle g_{1}, \cdots, g_{n}\right\rangle \in S\right.$ : $\left.\max _{j}\left\|g_{j}\right\|_{U}=A\right\}$. A normal families argument shows that $S_{A} \neq \varnothing$, and we see by the theorem that $S_{A}$ contains an element which is invariant under the group $\left\{\Phi_{\gamma}\right\}_{\gamma \in G}$.

We now turn to the more general situation. Let $R$ be a finite open Riemann surface embedded in the compact surface $R_{0}$ as indicated in the introduction. In addition to the algebra $H_{\infty}[R]$, it is convenient to introduce another algebra which we will denote by $\mathscr{A}[R]$. We define $\mathscr{A}[R]$ to be the set of those $f \in H_{\infty}[R]$ which are continuous on $\bar{R}$.

A result of Arens [4] is that the maximal ideal space of $\mathscr{A}[R]$ may be identified with $\bar{R}$ : Every complex homomorphism of $\mathscr{A}[R]$ is a point evaluation at some point of $\bar{R}$. This fact allows us to define certain distinguished subsets of $\mathfrak{M}[R]$ as follows. If $\phi \in \mathfrak{M}[R]$, then a fortiori $\phi$ acts as a complex homomorphism of 
$\mathscr{A}[R]$, and so there is a point $\alpha \in \widetilde{R}$ such that $\phi(f)=f(\alpha)$ for all $f \in \mathscr{A}[R]$. For each $\alpha \in \tilde{R}$, we define $\mathfrak{M}_{\alpha}[R]$ be to the set of all those $\phi \in \mathfrak{M}[R]$ such that $\phi(f)=f(\alpha)$ for all $f \in \mathscr{A}[R] ; \mathfrak{M}_{\alpha}[R]$ is called the fiber of $\mathfrak{M}[R]$ over $\alpha$. It is clearly a closed subset of $\mathfrak{M}[R]$.

We shall need to use the following nontrivial result on open Riemann surfaces: If $S$ is an open Riemann surface and if $z$ is any point of $S$, there is a function $f$ holomorphic on $S$ with a single, simple zero on $S$, this zero being at the point $z$. Note then that $1 / f$ is holomorphic on $S$ except at the point $z$ where it has a simple pole. For the existence of $f$, one may consult [5, Chapter 6].

We can now prove a pair of lemmas which generalize to our setting results known to hold in the case of the unit disc. The proofs do not differ greatly from the corresponding proofs in the disc case, but, for the sake of completeness, we include them.

Lemma 2.2. Let $f$ be in $H_{\infty}[R]$, and let $\alpha \in \partial R$. Let $\left\{\lambda_{n}\right\}$ be a sequence of points in $R$ such that $\lambda_{n} \rightarrow \alpha$. If $\lim f\left(\lambda_{n}\right)$ exists, say it is $\zeta$, then there exists $\phi \in \mathfrak{M}_{\alpha}[R]$ such that $\phi(f)=\zeta$.

Proof. Let $J=\left\{g: g \in H_{\infty}[R], \lim g\left(\lambda_{n}\right)=0\right\}$. The set $J$ is a closed ideal in $H_{\infty}[R]$, and so there is $\phi \in \mathfrak{M}[R]$ such that $\phi[J]=0$. We have $f-\zeta \in J$ so that $\phi(f)=\zeta$. To see that $\phi \in \mathfrak{M}_{\alpha}[R]$, note that if $g \in \mathscr{A}[R]$ is such that $g(\alpha)=0$ and $g(z) \neq 0$ for all $z \in \bar{R} \backslash\{\alpha\}$, then $\phi(g)=0$. q.e.d.

LEMma 2.3. If $f \in H_{\infty}[R]$ and if $\alpha \in \partial R$, then $\hat{f}$ is constant on $\mathfrak{M}_{\alpha}[R]$ if and only if $f$ is continuously extensible to $R \cup\{\alpha\}$.

Proof. Note that as usual, $\hat{f}$ is the function on $\mathfrak{M}[R]$ defined by $\hat{f}(\phi)=\phi(f)$. If $\hat{f}$ is constant on $\mathfrak{M}_{\alpha}[R]$, the preceding lemma implies that $f$ is so extensible. Conversely, suppose $f$ to be continuously extensible to $R \cup\{\alpha\}$. We may assume $f(\alpha)=0$. There exists a function $h \in \mathscr{A}[R]$ with the property that $h(\alpha)=1$ and $|h(z)|<1$ for $z \in \bar{R} \backslash\{\alpha\}$. The existence of such an $h$ follows from the result quoted from Behnke and Sommer: Let $\alpha$ lie in the component $\Gamma_{1}$ of $\partial R$, and let $\Delta_{1}$ be the component of $R_{0} \mid R$ which $\Gamma_{1}$ bounds. If $p_{0} \in \Delta_{1}$, then $R_{0} \backslash\left\{p_{0}\right\}$ is an open surface, and accordingly there is a function $h_{1}$ which is holomorphic on $R_{0} \backslash\left\{p_{0}\right\}$ save only for a single simple pole at a prescribed point $\beta$. If we locate $\beta$ sufficiently near $\alpha$ and in $R_{0} \backslash \bar{R}$ then we can find a neighborhood $N$ of $\alpha$ which includes $\beta$ and which has the property that for some constant $A,\left|h_{1}(z)\right| \leqq A$ on $R \backslash N$ while $\left|h_{1}(z)\right|>A$ on $N$ and the property that $h_{1}$ is one-to-one on $N$. It follows immediately from the Riemann mapping theorem that there is a function $g$ holomorphic on $h_{1}[R]$ and such that if $h(z)=g\left(h_{1}(z)\right)$, then $h$ has the desired property.

We now note that $\left(1-h^{n}\right) f$ converges to $f$ in $H_{\infty}[R]$. If $\phi \in \mathfrak{M}_{\alpha}$ we have by continuity that $\phi\left(\left(1-h^{n}\right) f\right) \rightarrow \phi(f)$, i.e., that $\lim \left(\phi\left(1-h^{n}\right) \phi(f)\right)=\phi(f)$. However, 
since $h$ is continuous at $\alpha$ and $h(\alpha)=1$, we have $\phi(h)=1$ so that $\phi\left(1-h^{n}\right)=0$ whence $\phi(f)=0$, and the result follows. q.e.d.

Before proceeding, we need to recall two results due to Behnke and Stein concerning a Cauchy formula on open Riemann surfaces. A convenient reference is [5].

THEOREM 2.4. Let the domain $G$ have compact closure in the noncompact Riemann surface $S$. Then for each $\zeta \in G$, there is a differential $d \omega(z, \zeta)$ which has a single pole and residue 1. This pole is of the first order, and it is located at $\zeta$. If $z \in G$ and if $V$ is a parametric disc about $z$ such that in $V$ the differential $d \omega(z, \zeta)$ is represented by the function $A_{V}(z, \zeta)$, then $A_{V}(z, \zeta)$ is, as a function of $\zeta$, a meromorphic function on $G$ which has only one pole and residue 1 . This pole is simple and is located at the point $z$.

We shall say that for a fixed $z$ and fixed choice of local coordinates, $d \omega(z, \zeta)$ is a meromorphic function of $\zeta$.

A consequence of the existence of this differential is a Cauchy formula for sets in open Riemann surfaces.

THEOREM 2.5. Let $G$ be a domain with compact closure in a noncompact Riemann surface, and let $d \omega(z, \zeta)$ be the elementary differential of the preceding theorem associated with $G$. Let $G^{*}$ be a domain such that $\bar{G}^{*} \subset G$ and such that $G^{*}$ is bounded by finitely many smooth curves. Then for every function $f$ which is holomorphic in $G^{*}$ and continuous on $\bar{G}^{*}$ we have, for every $\zeta \in G^{*}$,

$$
f(\zeta)=\frac{1}{2 \pi i} \int_{\partial G^{*}} f(z) d \omega(z, \zeta)
$$

The orientation on $\partial G^{*}$ is the positive orientation induced by the orientation in $G$.

Having the formula (2.1) for functions $f$ with continuous boundary values, we can show that the same formula obtains for functions which are merely in $H_{\infty}\left[G^{*}\right]$. Fix upon $\zeta \in G^{*}$, and let $f \in H_{\infty}\left[G^{*}\right]$. We have that $\partial G^{*}$ consists of finitely many smooth simple closed curves. Let $C$ be one of them, and let $C^{\prime}$ be another smooth simple closed curve which, together with $C$, bounds an annulus $A$ in $G^{*}$. We may choose $C^{\prime}$ so that $\zeta \notin \bar{A}$. We assert that

$$
\int_{C^{\prime}} f(z) d \omega(z, \zeta)=\int_{C} f(z) d \omega(z, \zeta)
$$

To see this, note that by the choice of the curve $C^{\prime}$, we can write $A=A_{1} \cup \cdots \cup A_{m}$ for some $m$ where each $A_{j}$ lies in a single parametric disc and where $\partial A_{j}$ consists of an arc in $C$, an arc in $C^{\prime}$, and a pair of smooth arcs in $A$ so that $A_{j}$ is a certain curvilinear quadrilateral. But then we have that 


$$
\int_{C^{\prime}} f(z) d \omega(z, \zeta)-\int_{C} f(z) d \omega(z, \zeta)=\sum_{j=1}^{m} \int_{\partial A_{j}} f(z) d \omega(z, \zeta) .
$$

Since $A_{j}$ lies in a single parametric disc, the integral $\int_{\partial A_{j}} f(z) d \omega(z, \zeta)$ can be represented as the integral round the unit circle of a function bounded and holomorphic in the unit disc, and so must be zero.

We can apply this process to each of the curves which make up the boundary of $G^{*}$ and conclude that

$$
\frac{1}{2 \pi i} \int_{\partial G^{*}} f(z) d \omega(z, \zeta)=\frac{1}{2 \pi i} \int_{\partial H^{*}} f(z) d \omega(z, \zeta)
$$

where $H^{*}$ is some domain such that $\zeta \in H^{*}$ and such that $\bar{H}^{*} \subset G^{*}$ and whose boundary consists of finitely many smooth simple closed curves. Since $f$ is continuous on $\bar{H}^{*}$, the latter integral is just $f(\zeta)$, and we may conclude that (2.1) is valid for functions which lie in $H_{\infty}\left[G^{*}\right]$.

We are now in a position to establish the fundamental result of this section.

THEOREM 2.6. Let $f_{1}, \cdots, f_{n} \in H_{\infty}[R]$, and let $\left|f_{1}(\zeta)\right|+\cdots+\left|f_{n}(\zeta)\right| \geqq \delta>0$ hold for all $\zeta \in R$. Then there exist $g_{1}, \cdots, g_{n} \in H_{\infty}[R]$ such that $\sum_{1}^{m} f_{j} g_{j}=1$.

Proof. For the duration of this proof, we shall denote $\mathfrak{M}[R]$ and $\mathfrak{M}_{\alpha}[R]$ simply by $\mathfrak{M}$ and $\mathfrak{M}_{\alpha}$ respectively. The space $\mathfrak{M}$ contains some obvious elements: if $\zeta \in R$, denote by $\phi_{\zeta}$ the element of $\mathfrak{M}$ given by $\phi_{\zeta}(f)=f(\zeta)$ for all $f \in H_{\infty}[R]$. Thus we have a map $\zeta \rightarrow \phi_{\zeta}$ from $R$ into $\mathfrak{M}$. Since $H_{\infty}[R]$ separates points on $R$, it follows easily that the map $\zeta \rightarrow \phi_{\zeta}$ is a homeomorphism of $R$ into $\mathfrak{M}$, and so $R$ may be regarded as a subset of $\mathfrak{M}$. It is known $[12$, p. 163] that the truth of our theorem is equivalent to the density of $R$ in $\mathfrak{M}$. Our proof consists of showing that $R$ is indeed dense in $\mathfrak{M}$.

Let the differential $d \omega(z, \zeta)$ be chosen in accordance with Theorem 2.4 where the domain $G$ of that theorem is replaced by a domain in $R_{0}$ which contains $\bar{R}$ in its interior. Thus if $f \in H_{\infty}[R]$, then

$$
f(\zeta)=\frac{1}{2 \pi i} \int_{\partial R} f(z) d \omega(z, \zeta)
$$

for all $\zeta \in R$. The topological structure of $R$ is such that there is, for each $j$, a $\Gamma_{j}^{\prime}$ which, together with $\Gamma_{j}$ bounds an annular subregion $A_{j}$ of $R$.

Let us consider the algebra $H_{\infty}\left[A_{1}\right]$. If $f \in H_{\infty}\left[A_{1}\right]$, and if $\zeta \in A_{1}$, we may write

$$
f(\zeta)=\frac{1}{2 \pi i} \int_{\partial A_{1}} f(z) d \omega(z, \zeta)
$$

Since $\partial A_{1}=\Gamma_{1}+\Gamma_{1}^{\prime}$, we obtain

$$
f(\zeta)=f_{1}(\zeta)+f_{2}(\zeta)
$$


where

$$
f_{1}(\zeta)=\frac{1}{2 \pi i} \int_{\Gamma_{1}} f(z) d \omega(z, \zeta)
$$

and

$$
f_{2}(\zeta)=\frac{1}{2 \pi i} \int_{\Gamma_{1}^{\prime}} f(z) d \omega(z, \zeta)
$$

Since for fixed $z \in \bar{R}$, and for a fixed choice of coordinates, $d \omega(z, \zeta)$ depends holomorphically on $\zeta$ except at $z$, we have that $f_{1}$ is holomorphic in the whole of $R$. Similarly, $f_{2}$ is continuous on $\Gamma_{1}$. It follows that both $f_{1}$ and $f_{2}$ are bounded, $f_{1}$ on $R$ and $f_{2}$ on $A_{1}$.

Let $\alpha \in \Gamma_{1}$, and let $\phi \in \mathfrak{M}_{\alpha}$. If $\mathfrak{M}_{\alpha}^{\prime}$ is the fiber in the maximal ideal space of $H_{\infty}\left[A_{1}\right]$ which lies over the point $\alpha$, we shall show that $\phi$ admits a unique extension to a complex homomorphism $\phi^{\prime}$ of $H_{\infty}\left[A_{1}\right]$, and that this extension is in $\mathfrak{M}_{\alpha}^{\prime}$.

We begin by showing that if $\phi^{\prime}$ is an extension of $\phi$ to a complex homomorphism of $H_{\infty}\left[A_{1}\right]$, then necessarily $\phi^{\prime} \in \mathfrak{M}_{\alpha}^{\prime}$. To say that $\phi^{\prime} \in \mathfrak{M}_{\alpha}^{\prime}$ is to say that if $f \in H_{\infty}\left[A_{1}\right]$ and if $f$ is continuous on $\bar{A}_{1}$, then $\phi^{\prime}(f)=f(\alpha)$. We know this relation to hold for all those $f$ which are continuous on $\bar{A}_{1}$ and are restrictions to $A_{1}$ of elements of $H_{\infty}[R]$. Suppose $\beta \in \bar{A}_{1} \mid\{\alpha\}$. Then there is a function $g \in \mathscr{A}[R]$ such that $g(\beta) \neq 0$ and $g(\alpha)=0$. We have that $\phi^{\prime}(g)=g(\alpha)=0$. Since $\phi^{\prime}$ acts on $\mathscr{A}\left[A_{1}\right]$ as point evaluation at some point of $\bar{A}_{1}$, it follows that $\phi^{\prime} \notin \mathfrak{M}_{\beta}^{\prime}$. The point $\beta$ is quite arbitrary, and so it must be that $\phi^{\prime} \in \mathfrak{M}_{\alpha}^{\prime}$.

Next, we see that there can be only one extension of $\phi$ to an element of $\mathfrak{M}_{\alpha}^{\prime}$. For if $\phi^{\prime}$ is an extension and if $f \in H_{\infty}\left[A_{1}\right]$, let us write $f=f_{1}+f_{2}$ in accordance with (2.4). By the linearity of $\phi^{\prime}$, we must have $\phi^{\prime}(f)=\phi^{\prime}\left(f_{1}\right)+\phi^{\prime}\left(f_{2}\right)$. Since $\phi^{\prime}$ is an extension of $\phi$ and $f_{1} \in H_{\infty}[R]$, we have $\phi^{\prime}\left(f_{1}\right)=\phi\left(f_{1}\right)$. Also, since $f_{2}$ is continuous at $\alpha, \phi^{\prime}\left(f_{2}\right)=f_{2}(\alpha)$. Thus we have

$$
\phi^{\prime}(f)=\phi\left(f_{1}\right)+f_{2}(\alpha) .
$$

It follows that there is at most one extension of $\phi$ to an element of $\mathfrak{M}_{\alpha}^{\prime}$.

Finally, if $\phi \in \mathfrak{M}_{\alpha}$, and if, for every $f \in H_{\infty}\left[A_{1}\right], \phi^{\prime}(f)$ is defined by (2.5), then $\phi^{\prime}$ is an extension of $\phi$ to an element of $\mathfrak{M}_{\alpha}^{\prime}$. We first show that $\phi^{\prime}$ is an extension of $\phi$. To see this, suppose that $f \in H_{\infty}[R]$. Then also $f \in H_{\infty}\left[A_{1}\right]$, and for $\zeta \in A_{1}$, we have the decomposition (2.4). In this decomposition, $f$ and $f_{1}$ are both in $H_{\infty}[R]$, so it follows that $f_{2}$ is also in $H_{\infty}[R]$. Since $f_{2}$ is continuous on $\Gamma_{1}$, we have $\phi^{\prime}(f)=\phi\left(f_{1}\right)+f_{2}(\alpha)=\phi\left(f_{1}\right)+\phi\left(f_{2}\right)=\phi\left(f_{1}+f_{2}\right)=\phi(f)$, and so $\phi^{\prime}$ is an extension of $\phi$.

It remains only to show that $\phi^{\prime}$ is multiplicative on $H_{\infty}\left[A_{1}\right]$ since it is plainly linear. In addition to $f$, suppose given $g \in H_{\infty}\left[A_{1}\right]$, and write $g=g_{1}+g_{2}$ in accordance with (2.4). The linearity of $\phi^{\prime}$ yields

$$
\phi^{\prime}(f g)=\phi^{\prime}\left(f_{1} g_{1}\right)+\phi^{\prime}\left(f_{1} g_{2}\right)+\phi^{\prime}\left(f_{2} g_{1}\right)+\phi^{\prime}\left(f_{2} g_{2}\right) \text {. }
$$


Since $f_{1} g_{1}$ is in $H_{\infty}[R]$, and since $\phi^{\prime}$ is an extension of $\phi$, we have that $\phi^{\prime}\left(f_{1} g_{1}\right)=\phi\left(f_{1} g_{1}\right)=\phi\left(f_{1}\right) \phi\left(g_{1}\right)$. Consider next the term $\phi^{\prime}\left(f_{2} g_{2}\right)$. Write $f_{2} g_{2}=h_{1}+h_{2}$ by (2.4). Since $f_{2} g_{2}$ and $h_{2}$ are both continuous at $\alpha$, so is $h_{1}$, and we have $\phi^{\prime}\left(f_{2} g_{2}\right)=\phi\left(h_{1}\right)+h_{2}(\alpha)=h_{1}(\alpha)+h_{2}(\alpha)=f_{2}(\alpha) g_{2}(\alpha)$.

Finally, we consider the term $f_{1} g_{2}$. We shall show that $\phi^{\prime}\left(f_{1} g_{1}\right)=\phi\left(f_{1}\right) g_{2}(\alpha)$ by proving that

$$
\phi^{\prime}\left(f_{1} g_{2}-f_{1} g_{2}(\alpha)\right)=0 .
$$

To do this, write $f_{1} g_{2}-f_{1} g_{2}(\alpha)=h_{1}+h_{2}$ by (2.4). Then since $g_{2}$ is continuous at $\alpha$, it follows that $f_{1} g_{2}-f_{1} g_{2}(\alpha)$ is also continuous at $\alpha$. Accordingly, we have

$$
\begin{aligned}
\phi^{\prime}\left(f_{1} g_{2}-f_{1} g_{2}(\alpha)\right) & =\phi\left(h_{1}\right)+h_{2}(\alpha) \\
& =h_{1}(\alpha)+h_{2}(\alpha) \\
& =0 .
\end{aligned}
$$

The term $f_{2} g_{1}$ is treated in a similar fashion and we conclude that every $\phi \in \mathfrak{M}_{\alpha}$ admits a unique extension to an element of $\mathfrak{M}_{\alpha}^{\prime}$.

We can now prove that every element $\phi$ of $\mathfrak{M}_{\alpha}$ is the weak* limit of point evaluations at points in $R$. For let $\phi^{\prime}$ be the unique extension of $\phi$ to an element of $\mathfrak{M}_{\alpha}^{\prime}$. By virtue of Theorem 2.1, there is a net $\left\{\phi_{\beta}^{\prime}\right\}$ of point evaluations at points in $A_{1}$ such that $\phi_{\beta}^{\prime} \rightarrow \phi^{\prime}$ in the weak* topology on the maximal ideal space of $H_{\infty}\left[A_{1}\right]$. But then it is immediate that if $\phi_{\beta}$ is the restriction of $\phi_{\beta}^{\prime}$ to $H_{\infty}[R]$ then $\left\{\phi_{\beta}\right\}$ converges to $\phi$. Note that by invoking Theorem 2.1 we are making use of Carleson's result for the disc, Theorem 1.1.

Let us next consider a point $\alpha \in R$ (i.e., $\alpha \in \bar{R} \backslash \partial R$ ). We observe that the associated $\mathfrak{M}_{\alpha}$ consists of a single complex homomorphism. To see this, let $f_{0} \in \mathscr{A}[R]$ have a single zero on $\bar{R}$, that a simple zero at $\alpha$. Consider $f \in H_{\infty}[R]$ such that $f(\alpha)=0$. Then $f / f_{0} \in H_{\infty}[R]$, and we have, for each $\phi \in \mathfrak{M}_{\alpha}$

$$
\phi(f)=\phi\left(f / f_{0}\right) \phi\left(f_{0}\right) .
$$

But $\phi\left(f_{0}\right)=0$ since $\phi \in \mathfrak{M}_{\alpha}$ and so $\phi(f)=0$ if $f(\alpha)=0$. This means that $\phi$ acts on $H_{\infty}[R]$ as evaluation at $\alpha$.

Recalling that if $\zeta \in R$, then $\phi_{\zeta}$ is point evaluation at $\zeta$ and that the map $\zeta \rightarrow \phi_{\zeta}$ has been observed to be a homeomorphism of $R$ into $\mathfrak{M}$, we see that we have shown $R$ to be dense in $\mathfrak{M}$. q.e.d.

We can obtain a bit of information about the structure of the fiber $\mathfrak{M}_{\alpha}$ from the proof of Theorem 2.6. The map $1: f \rightarrow f \mid A_{1}$ defines an isomorphism of $H_{\infty}[R]$ into $H_{\infty}\left[A_{1}\right]$ and this isomorphism has the adjoint $\imath^{*}: \mathfrak{M}\left[A_{1}\right] \rightarrow \mathfrak{M}[R]$. Our proof has shown that if $\alpha \in \Gamma_{1}$, then $\imath^{*}$ carries $\mathfrak{M}_{\alpha}\left[A_{1}\right]$ homeomorphically onto $\mathfrak{M}_{\alpha}[R]$. Since $H_{\infty}\left[A_{1}\right]$ has a great number of automorphisms, it is very easily seen that if $\alpha$ and $\beta$ are points of $\partial A_{1}$, then $\mathfrak{M}_{\alpha}\left[A_{1}\right]$ and $\mathfrak{M}_{\beta}\left[A_{1}\right]$ are homeomorphic. We next 
note that the structure of $\mathfrak{M}_{\alpha}\left[A_{1}\right]$ is independent of the conformal structure of $A_{1}$. This is so for if we take for $R$ the open unit disc and for $A$ some annulus with outer boundary $\Lambda$, our proof shows that $\mathfrak{M}_{1}[A]$ is homeomorphic to $\mathfrak{M}_{1}[U]$. This observation is independent of the conformal structure of $A$. Thus, we have the following result:

Corollary 2.7. If $\alpha \in \partial R$, then $\mathfrak{M}_{\alpha}[R]$ is homeomorphic to $\mathfrak{M}_{1}[U]$.

We may therefore conclude that the fibers in $\mathfrak{M}[R]$ which lie over points of $\partial R$ are all homeomorphic and that their structure is independent of the particular choice of $R$. This is to be expected, since it seems "obvious" that the structure of an $\mathfrak{M}_{\alpha}[R]$ depends only on the behaviour near $\alpha$ of the functions in $H_{\infty}[R]$, and this is a purely local matter.

3. Uniformization and $\mathfrak{M}[R]$. We now turn to the consideration of another aspect of the relation between the two spaces $\mathfrak{M}[U]$ and $\mathfrak{M}[R]$, viz., that relation induced by the uniformizing map from $U$ onto $R$. The surface $R$ carries nonconstant bounded holomorphic functions, and so its universal covering surface is conformally equivalent to the disc.

Let $F: U \rightarrow R$ be a uniformizing map, and let $G$ be the group of conformal maps of $U$ onto itself under which $F$ is invariant. Recall that the point $z_{0} \in \bar{U}$ is said to be a limit point of the group $G$ if it is a limit point of the set $\{\gamma(z)\}_{\gamma \in G}$ for some $z \in U$. The set of limit points of the group $G$ is a closed set of measure zero in the unit circle (for this point, see Tsuji [19, p. 525]), and the embedding of $R$ in the surface $R_{0}$ is such that the function $F$ admits analytic continuation across the complement in the unit circle of the set of limit points of $G$.

The map $F$ induces a homomorphism $\Phi: H_{\infty}[R] \rightarrow H_{\infty}[U]$ by means of $(\Phi f)(z)=f(F(z))$ for all $z \in U$ and all $f \in H_{\infty}[R]$. In its turn, the homomorphism $\Phi$ induces a continuous map $\Phi^{*}: \mathfrak{M}[U] \rightarrow \mathfrak{M}[R]$, and it is this map which we propose to study.

In order to establish our result, we shall need to make use of a result of Ahlfors [1]. (For an alternative proof, one may consult Royden [15].)

THEOREM 3.1. There exists a function $P$ holomorphic on a neighborhood of $\bar{R}$ which maps $R$ onto the open unit disc in an one-to-one manner for some $n$ and which satisfies $|P|=1$ on $\partial R$.

In our later work, we shall have to make a great deal of use of the function $P$. For the present, we use it to establish the chief result of the present section:

THEOREM 3.2. If $\alpha_{0} \in \bar{U}$ is not a limit point of the group $G$, and if $\beta_{0}=F\left(\alpha_{0}\right)$, then $\Phi^{*}$ maps the fiber $\mathfrak{M}_{\alpha_{0}}[U]$ homeomorphically onto the fiber $\mathfrak{M}_{\beta_{0}}[R]$. 
Before beginning the proof of this theorem, it is convenient to establish a rather technical lemma.

LEMMA 3.3. Let $V$ be a simply connected subdomain of $U$ whose boundary contains the arc $\lambda$ of $\Lambda$, the unit circle. Let $\mathrm{\imath}: H_{\infty}[U] \rightarrow H_{\infty}[V]$ be the restriction map and let $\mathrm{\imath}^{*}$ be its adjoint. Let $\alpha_{1}$ be some point of $\lambda$. Then $\mathrm{\imath}^{*}$ maps $\mathfrak{M}_{\alpha_{1}}[V]$ homeomorphically onto $\mathfrak{M}_{\alpha_{1}}[U]$.

Proof. We remark that $\imath^{*}$ is fiber preserving in that if $\alpha \in \bar{V}$ and $\phi \in \mathfrak{M}_{\alpha}[V]$, then $\mathrm{\imath}^{*} \phi \in \mathfrak{M}_{\alpha}[U]$. Thus, $\mathrm{\imath}^{*}$ takes $\mathfrak{M}_{\alpha_{1}}[V]$ into $\mathfrak{M}_{\alpha_{1}}[U]$. The result of Carleson on the corona problem for the simply connected case together with the fiber preserving property of $\imath^{*}$ shows that $\imath^{*}$ carries $\mathfrak{M}_{\alpha_{1}}[V]$ onto $\mathfrak{M}_{\alpha_{1}}[U]$. It remains to show that $\imath^{*}$ is one-to-one on $\mathfrak{M}_{\alpha_{1}}[V]$. We shall establish this point by showing that $H_{\infty}[U]$ separates points in the fiber $\mathfrak{M}_{\alpha_{1}}[V]$.

Let $\phi, \psi \in \alpha_{1} \mathfrak{M}[V]$. There exists $f \in H_{\infty}[V]$ such that $\phi(f)=0, \psi(f)=1$. Write $f=u+i v$ where $u$ and $v$ are harmonic, bounded, real valued functions. Let us denote by $k$ a conformal map of $U$ onto $V$. Let $\lambda^{\prime}$ be the inverse image of $\lambda$ under $k$; the function $k$ is then continued across the interior of $\lambda^{\prime}$.

The function $u$ has $L_{\infty}$ boundary values a.e. on $\lambda$. Let $u_{1}$ be a function harmonic in $U$ with boundary values 0 off $\lambda$ and boundary values equal to those of $u$ on $\lambda$. We have that $u=\left(u-u_{1}\right)+u_{1}$ in $V$.

The function $h=\left(u-u_{1}\right) \circ k$ is a function harmonic and bounded in $U$, and it has boundary values 0 a.e. on $\lambda^{\prime}$. Consider the function $f_{1}$ given by

$$
f_{1}(z)=\frac{1}{2 \pi i} \int_{-\pi}^{\pi} \frac{e^{i \theta}+z}{e^{i \theta}-z} h\left(e^{i \theta}\right) d \theta .
$$

Since $h=0$ a.e. on $\lambda^{\prime}$, we have that

$$
f_{1}(z)=\frac{1}{2 \pi} \int_{\lambda^{\prime} c} \frac{e^{i \theta}+z}{e^{i \theta}-z} h\left(e^{i \theta}\right) d \theta
$$

and, consequently, the function $f_{1}$ is continued across the interior of $\lambda^{\prime}$. Note that the real part of $f_{1}$ is $h$.

Let $v_{1}$ be the harmonic conjugate of $u_{1}$ which vanishes at 0 , and let $f_{2}=f_{1} \circ k^{-1}$. Consider then the function:

$$
g=\exp \left(f_{2}+\left(u_{1}+i v_{1}\right)\right)=\left(\exp f_{2}\right)\left(\exp \left(u_{1}+i v_{1}\right)\right) \text {. }
$$

We have $g \in H_{\infty}[V]$, and we also see that:

$$
\phi(g)=\exp \left(\phi\left(f_{2}\right)+\phi\left(u_{1}+i v_{1}\right)\right)=\exp \phi(f)=1 \text {. }
$$

Similarly, $\psi(g)=e$. On the other hand, we have:

$$
\begin{aligned}
& \phi(g)=\phi\left(\exp f_{2}\right) \phi\left(\exp \left(u_{1}+i v_{1}\right)\right) \\
& \psi(g)=\psi\left(\exp f_{2}\right) \psi\left(\exp \left(u_{1}+i v_{1}\right)\right)
\end{aligned}
$$

The function $f_{2}$ is, by definition, $f_{1} \circ k^{-1}$. Since $f_{1}$ is continued across the interior of $\lambda^{\prime}$ and $k^{-1}$ across the interior of $\lambda$, it follows that $f_{2}$ is holomorphic in a 
neighborhood of $\alpha_{1}$, and hence that $\phi\left(\exp f_{2}\right)=\psi\left(\exp f_{2}\right)=\exp f_{2}\left(\alpha_{1}\right)$. Consequently, $\phi\left(\exp \left(u_{1}+i v_{1}\right)\right) \neq \psi\left(\exp \left(u_{1}+i v_{1}\right)\right)$. Since $\exp \left(u_{1}+i v_{1}\right)$ is in $H_{\infty}[U]$, we have our lemma.

Proof of Theorem 3.2. As we have already observed, there is a natural embedding of $U$ in $\mathfrak{M}[U]$ and of $R$ in $\mathfrak{M}[R]$. In the sense of these embeddings, the map $\Phi^{*}$ is plainly an extension of the map $F$. Since $R$ is dense in $\mathfrak{M}[R]$, since $\mathfrak{M}[U]$ is compact, and since $F$ maps $U$ onto $R$, it follows immediately that $\Phi^{*}$ maps $\mathfrak{M}[U]$ onto $\mathfrak{M}[R]$.

The fibers in $\mathfrak{M}[U]$ which lie over points of $U$ are trivial as are those of $\mathfrak{M}[R]$ which lie over points of $R$. Accordingly, we may confine our attention to the action of $\Phi^{*}$ on fibers $\mathfrak{M}_{\alpha_{0}}[U]$ for which $\alpha_{0}$ lies in the unit circle.

If $\alpha_{0}$ lies on the unit circle and is not a limit point of the group $G$, then the point $\beta_{0}=F\left(\alpha_{0}\right)$ lies on $\partial R$. We assert that $\Phi^{*}$ carries $\mathfrak{M}_{\alpha_{0}}[U]$ into $\mathfrak{M}_{\beta_{0}}[R]$. To establish this point, let $\phi \in \mathfrak{M}_{\alpha_{0}}[U]$. As we have observed in Lemma 2.3, there is a function $h$ in the algebra $\mathscr{A}[R]$ with the property that $|h(z)|<1$ for $z \in \bar{R} \backslash\left\{\beta_{0}\right\}$ but also with the property that $h\left(\beta_{0}\right)=1$. Then $\left(\Phi^{*} \phi\right)(h)=\phi(h \circ F)$. Since $F$ is continuous at $\alpha_{0}, \phi(h \circ F)=h\left(F\left(\alpha_{0}\right)\right)=1$. Since $|h(z)|<1$ for all $z \in \bar{R}$ except for $z=\beta_{0}$, and since $h \in \mathscr{A}[R]$, we conclude that $\Phi^{*} \phi \in \mathfrak{M}_{\beta_{0}}[R]$.

Let $P: R \rightarrow U$ be the mapping guaranteed by Theorem 3.1. Thus $P$ maps $\partial R$ onto $\Lambda$ and is continued across $\partial R$. From $P$ we derive a homomorphism $\Psi: H_{\infty}[U] \rightarrow H_{\infty}[R]$ by means of $\Psi f=f \circ P$. Denote by $\Psi^{*}$ the adjoint of $\Psi$. The composition $P \circ F$ carries $U$ onto itself and the function $P \circ F$ is continued across an arc of $\Lambda$ which contains the point $\alpha_{0}$. Let $N$ be a neighborhood of $\alpha_{0}$ in $\bar{U}$ on which $P \circ F$ is analytic and one-to-one, and let $M=P \circ F[N]$. Set $\gamma_{0}=P\left(F\left(\alpha_{0}\right)\right)=P\left(\beta_{0}\right)$. For the sake of notation, we set $T=P \circ F \mid N$. As with $P$ and $F$, we let $\Theta: H_{\infty}[M] \rightarrow H_{\infty}[N]$ be given by $\Theta f=f \circ T$, and we have $\Theta^{*}: \mathfrak{M}[N] \rightarrow \mathfrak{M}[M]$. Since $T$ is a one-to-one conformal map of $N$ onto $M$, $\Theta$ is an isometric isomorphism, and it follows that $\Theta^{*}$ is a homeomorphism whose range is all of $\mathfrak{M}[M]$.

Let $\imath: H_{\infty}[U] \rightarrow H_{\infty}[N]$ be the restriction map, and let $\imath^{*}: \mathfrak{M}[N] \rightarrow \mathfrak{M}[U]$ be the adjoint of $\mathrm{t}$. Similarly, let $\kappa: H_{\infty}[U] \rightarrow H_{\infty}[M]$ be the restriction map with adjoint $\kappa^{*}$. Between the various maps we have defined we have the relation $\imath \circ \Phi \circ \Psi=\Theta \circ \kappa$ whence the relation

$$
\Psi^{*} \circ \Phi^{*} \circ \imath^{*}=\kappa^{*} \circ \Theta^{*} .
$$

We have observed that $\Theta^{*}$ is a homeomorphism onto, and it is clear that it takes the fiber over $\alpha_{0}$ in $\mathfrak{M}[N]$ onto the fiber over $\gamma_{0}$ in $\mathfrak{M}[M]$. Lemma 3.3 implies that $\imath^{*}$ maps $\mathfrak{M}_{\alpha_{0}}[N]$ homeomorphically onto $\mathfrak{M}_{\alpha_{0}}[U]$ and that $\kappa^{*}$ maps $\mathfrak{M}_{\gamma_{0}}[M]$ homeomorphically onto $\mathfrak{M}_{\gamma_{0}}[U]$.

The commutativity relation (3.1) now allows the following conclusions: (1) $\Phi^{*}$ is necessarily one-to-one on $\mathfrak{M}_{\alpha_{0}}[U],(2) \Psi^{*}$ is one-to-one on the range of $\Phi^{*}$, and (3) $\Psi^{*}$ maps $\mathfrak{M}_{\beta_{0}}[U]$ onto $\mathfrak{M}_{\gamma_{0}}[U]$. 
In order to conclude the proof of our theorem, we need only prove that $\Phi^{*}$ maps $\mathfrak{M}_{\alpha_{0}}[U]$ onto $\mathfrak{M}_{\beta_{0}}[R]$. Our remarks so far have shown in effect that if $N_{0}$ is some small open set in $U$ whose Euclidean closure contains an arc of $\Lambda$ which contains $\alpha_{0}$, then $\Phi^{*}$ maps $N_{0}^{-}$, the closure operator ${ }^{-}$here referring to closure in $\mathfrak{M}[U]$, onto $\Phi^{*}\left[N_{0}^{-}\right]$in a homeomorphic way. (Remark that because of the nature of the weak* topology in $\mathfrak{M}[U]$, this latter assertion differs from saying that $\Phi^{*}$ acts as local homeomorphism, i.e., that $\Phi^{*}$ acts as a homeomorphism on some neighborhood of each point in its domain. This difference can be made explicit by observing that there are points in $\mathfrak{M}_{\alpha_{0}}$ which have weak ${ }^{*}$ neighborhoods which meet no fiber $\mathfrak{M}_{\alpha}$ for $|\alpha|=1$ other than $\mathfrak{M}_{\alpha_{0}}$. For instance, if $g(z)=\exp \left(\left(z+\alpha_{0}\right) /\left(z-\alpha_{0}\right)\right)$, then $g \in H_{\infty}[U], g$ is continuous on $\bar{U} \backslash\left\{\alpha_{0}\right\}$, and $|g|=1$ on $\partial U \backslash\left\{\alpha_{0}\right\}$. The set $\left\{\phi \in \mathfrak{M}[U]:|\hat{g}(\phi)|<\frac{1}{2}\right\}$ is a weak* open set which contains no point of any $\mathfrak{M}_{\alpha}$ other than $\mathfrak{M}_{\alpha_{0}}$.)

If $\phi_{0} \in \mathfrak{M}_{\beta_{0}}[R]$, the density of $R$ in $\mathfrak{M}[R]$ yields a net $\left\{\phi_{\alpha}: \alpha \in D\right\}$ of points of $R$ such that $\phi_{\alpha} \rightarrow \phi_{0}$. Choosing $N_{0}$ as indicated in the last paragraph, we can determine points $\phi_{\alpha}^{\prime} \in \mathfrak{M}[U]$ by the condition that $\Phi^{*}\left(\phi_{\alpha}^{\prime}\right)=\phi_{\alpha}$ and $\phi_{\alpha}^{\prime} \in N_{0}$. The latter condition may allow us to find such $\phi_{\alpha}^{\prime}$ only for a subset of the $\alpha$ 's, but if $D_{1}$ is the set of all the $\alpha$ for which such a determination of $\phi_{\alpha}^{\prime}$ is possible, then $D \backslash D_{1}$ will be finite; we may as well assume $D=D_{1}$. But then since $\Phi^{*}$ is a homeomorphism on $N_{0}^{-} \subset \mathfrak{M}[U]$, the net $\left\{\phi_{\alpha}^{\prime}: \alpha \in D\right\}$ will converge, say to $\phi_{0}^{\prime}$, and we shall have $\Phi^{*}\left(\phi_{0}^{\prime}\right)=\phi_{0}$. Note finally that $\phi_{0}^{\prime} \in \mathfrak{M}_{\alpha_{0}}[U]$, and our theorem follows.

There is an immediate corollary of the proof just given. We have noted that $\Psi^{*}$ is one-to-one on the range of $\Phi^{*}$ and that $\Psi^{*}$ maps $\mathfrak{M}_{\beta_{0}}[R]$ onto $\mathfrak{M}_{\gamma_{0}}[U]$. Consequently, we have the following fact:

COROLLARY 3.4. $\Psi^{*}$ maps $\mathfrak{M}_{\beta_{0}}[R]$ homeomorphically onto $\mathfrak{M}_{\gamma_{0}}[U]$.

This fact has been noticed by Alling [3] who obtained it in the course of his proof of the corona conjecture on finite Riemann surfaces, and not as a consequence thereof.

4. An example. Our Theorem 3.2 can be used to obtain a rather curious result about the map $\Phi^{*}$. In Theorem 3.2 , we are concerned exclusively with the behaviour of the map $\Phi^{*}$ on fibers $\mathfrak{M}_{\alpha_{0}}[U]$ which lie over points of $\Lambda$ which are not limit points of the group $G$. It is reasonable to inquire about the behaviour of $\Phi^{*}$ on fibers which lie over limit points of the group: We shall see that the behaviour of $\Phi^{*}$ on these fibers is indeed complicated.

Consider the special case that $R$ happens to be an annulus $A$ bounded by analytic curves. If $F, G, \Phi$, and $\Phi^{*}$ are as in Theorem 3.2 , we can normalize $G$ so that the sole limit points of $G$ are the points $i$ and $-i$. Further, we can choose for a generator of $G$ an element $\gamma$ such that for any $z_{0} \in U, \gamma^{n}\left(z_{0}\right) \rightarrow i$ as $n \rightarrow+\infty$. The element $\gamma$ of $G$ induces an automorphism of $H_{\infty}[U]$ and so can be extended 
in a natural way to a homeomorphism $\gamma^{*}$ of $\mathfrak{M}[U]$ onto itself. Observe that if $f \in H_{\infty}[A]$, then the function $(\Phi f)^{\wedge} \in \hat{H}_{\infty}[U]$ is invariant under $\gamma^{*}$.

Choose a point $\alpha_{0} \in \Lambda$, and let $\beta_{0}=F\left(\alpha_{0}\right)$. Then by the theorem $\Phi^{*}$ maps $\mathfrak{M}_{\alpha_{0}}[U]$ homeomorphically onto $\mathfrak{M}_{\beta_{0}}[A]$. Since $F$ is invariant under $G$ and $\Phi^{*}$ is an extension of $F$, it follows that $\Phi^{*}$ is also invariant under the group of homeomorphisms of $\mathfrak{M}[U]$ generated by $\gamma^{*}$. It follows in particular that each of the fibers $\mathfrak{M}_{\alpha_{n}}[U]$ is carried onto $\mathfrak{M}_{\beta_{0}}[A]$ if $\alpha_{n}=\gamma^{n}\left(\alpha_{0}\right)$ for $n=0,1,2, \cdots$.

Fix a point $\phi_{0} \in \mathfrak{M}_{\beta_{0}}[A]$, and determine $\psi_{0} \in \mathfrak{M}_{\alpha_{0}}[U]$ by $\Phi^{*}\left(\psi_{0}\right)=\phi_{0}$. Then $\Phi^{*}$ takes every point of the set $\left\{\gamma^{* n}\left(\psi_{0}\right)\right\}^{-}=S$ onto $\phi_{0}$. For ease of notation, set $\psi_{n}=\gamma^{* n}\left(\psi_{0}\right)$. We see that the limit points of the set $S$ all lie in the fiber $\mathfrak{M}_{i}[U]$. In order to study the set $S$, it is convenient to prove a lemma.

LemMA 4.1. Let $\left\{z_{n}\right\}_{n=1}^{\infty}$ be a set of points in $\Lambda$ whose sole cluster point is 1 and which satisfy $1 \neq z_{n}$ for all $n$. Let $\left\{\alpha_{n}\right\}_{n=1}^{\infty}$ be a bounded set of complex numbers. There exists a function $f \in H_{\infty}[U]$ such that $f$ is continuous on $\bar{U} \backslash\{1\}$, and $f\left(z_{n}\right)=\alpha_{n}$.

Proof. The set $\left\{z_{n}\right\}_{n=1}^{\infty} \cup\{1\}$ is a closed set of measure zero, and so by a theorem of Rudin $\left[12\right.$, p. 81], we can find a function $h_{n} \in \mathscr{A}[U]$ such that $\left\|h_{n}\right\|_{U}=1$, $h_{n}\left(z_{n}\right)=1$, and $h_{n}\left(z_{m}\right)=0$ for $m \neq n$. In addition, we can require that $\left|h_{n}(z)\right|<1$ if $z \in \bar{U}, z \neq z_{n}$. Define $f$ by means of

$$
f(z)=\sum_{n=1}^{\infty} \alpha_{n}\left(h_{n}(z)\right)^{m(n)}
$$

where $m(n)$ is, for each integer $n$, an integer chosen as follows. For each $n$, let $D_{n}$ be a small neighborhood of $z_{n}$ in $\bar{U}$ such that for all $m$ and $n, \bar{D}_{n} \cap \bar{D}_{m}=\varnothing$ unless $m=n$. Since $\left|h_{n}(z)\right|<1$ if $z \neq z_{n}$, we can choose $m(n)$ so that $\left|h_{n}(z)\right|^{m(n)}$ is less than $2^{-n}$ for $z \notin D_{n}$. With these choices of $m(n)$, the series defining $f$ converges uniformly on compact sets in $\bar{U} \backslash\{1\}$ to an element of $H_{\infty}[U]$ which is continuous on $\bar{U} \backslash\{1\}$ and which takes the value $\alpha_{n}$ at the point $z_{n}$.

The lemma implies that if $g$ is any bounded, complex valued function defined on the set $\left\{\psi_{n}\right\}_{n \geqq 0}$, then there is $f \in H_{\infty}[U]$ such that $\hat{f}\left(\psi_{n}\right)=g\left(\alpha_{n}\right)$ for $n=0,1,2, \cdots$. Thus, every bounded complex valued function on $\left\{\psi_{n}\right\}_{n \geq 0}$ admits an extension to a function continuous on $\mathfrak{M}[U]$, and so, a fortiori, to a function continuous on the set $S$. Since $S$ is a compact space which contains $\left\{\psi_{n}\right\}_{n \geqq 0}$ as a dense subset, it follows that $S$ is topologically $\beta\left(\left\{\psi_{n}\right\}_{n \geqq 0}\right), \beta$ here indicating the Čech compactification.

Since the set $\left\{\psi_{n}\right\}_{n \geqq 0}$ is a discrete subset of $\mathfrak{M}[U]$ of cardinality $\aleph_{0}$, it follows that $S$ is of cardinality $2^{\mathrm{c}}$ (cf. $[10$, p. 130]). We may therefore conclude that the point $\phi_{0}$ is the image of $2^{\mathfrak{c}}$ distinct points in $\mathfrak{M}_{i}[U]$. Remark that the point $\phi_{0}$ was chosen in a completely arbitrary fashion within the fiber $\mathfrak{M}_{\beta_{0}}[A]$, and also 
that the fiber $\mathfrak{M}_{\beta_{0}}[A]$ was chosen arbitrarily. We may summarize these results as follows:

THEOREM 4.2. The function $\Phi^{*}$ maps $\mathfrak{M}_{i}[U]$ into $\mathfrak{M}[A]$ in such a way that every point in every fiber $\mathfrak{M}_{\beta}[A]$ for $\beta \in \partial A$ has $2^{c}$ preimages in $\mathfrak{M}_{i}[U]$.

5. The interpolation problem. In this section, we obtain a characterization of interpolation sets on finite open Riemann surfaces. We begin with a definition.

Definition. Let $R$ be a Riemann surface, and let $S$ be a set in $R$. For every $a \in S$ we define $d_{R}(a ; S)$ by means of $d_{R}(a ; S)=\sup |f(a)|$ where the sup is extended over all $f$ in $H_{\infty}[R]$ which vanish on the set $S \backslash\{a\}$ and which satisfy $\|f\|_{R} \leqq 1$.

The function $d_{R}(; S)$ is a decreasing function of the domain $R$ in the following sense: Suppose $R^{\prime}$ to be a surface which contains $R$, and let $S \subset R$. Then $S \subset R^{\prime}$, and we can compute $d_{R^{\prime}}(a ; S)$ as well as $d_{R}(a ; S)$ if $a \in S$. Since $R^{\prime} \supset R$, we have $d_{R^{\prime}}(a ; S) \leqq d_{R}(a ; S)$. Note also that $d_{R}(; S)$ is conformally invariant: If $F$ is a conformal (one-to-one) mapping of $R$ onto $R^{\prime}$, then $d_{R}(a ; S)=d_{R^{\prime}}(F(a), F[S]$ ).

A close relationship exists between the function $d_{R}(; S)$ and the interpolation problem for the set $S$. This relationship is already to be seen in the case of the unit disc as is shown by the following lemma.

Lemma 5.1. Let $S=\left\{z_{n}\right\}_{n=1}^{\infty}$ be a set in $U$. Then

$$
d_{U}\left(z_{n} ; S\right)=\prod_{k=1 ; k \neq n}^{\infty}\left|\frac{z_{n}-z_{k}}{1-\bar{z}_{k} z_{n}}\right| .
$$

Proof. Consider the Blaschke product $B_{n}$ given by

$$
B_{n}(z)=\prod_{k=1}^{\infty} \frac{\bar{z}_{k}}{\left|z_{k}\right|} \frac{z-z_{k}}{1-\bar{z}_{k} z} .
$$

It could happen that the product defining $B_{n}$ diverges to zero, but if this occurs for any given value of $n$, then it also occurs for all other values of $n$. If all the $B_{n}$ reduce to zero, then we have, for each $z_{n}, d_{v}\left(z_{n} ; S\right)=0$, and the lemma is verified, though in an uninteresting way.

Suppose then that none of the $B_{n}$ reduce to zero. A normal families argument yields a function $f$ such that $f\left(z_{k}\right)=0$ if $k \neq n$, which satisfies $\|f\|_{U} \leqq 1$, and which is such that $\left|f\left(z_{n}\right)\right|=d_{U}\left(z_{n} ; S\right)$. Since $B_{n}$ satisfies $\left\|B_{n}\right\|_{U} \leqq 1$ and $B_{n}\left(z_{k}\right)=0$ for $k \neq n$, it follows that $\left|f\left(z_{n}\right)\right| \geqq\left|B_{n}\left(z_{n}\right)\right|$. We assert that the reversed inequality holds also. This is seen by writing $f=B E F$ in accordance with the factorization theorem so that $B$ is a Blaschke product, $E$ is a singular function, and $F$ is the outer part of $f$. (For definitions of singular and outer functions, together with a discussion of the factorization theorem, see [12].) Since $E$ and $F$ are both zero free, the zeros of $B$ include those of $B_{n}$, and so for all $z \in U$, we have 


$$
|B(z)| \leqq\left|B_{n}(z)\right| .
$$

We shall have strict inequality unless the zeros of $B$ coincide with those of $B_{n}$, i.e., unless $B=B_{n}$. We also have that for $z \in U,|E(z)| \leqq 1$ with strict inequality unless $E$ is constant. Similarly, $|F(z)| \leqq 1$, and we have strict inequality unless $F$ is constant. From these observations, it follows that $f=c B_{n}$ where $c$ is some complex number of absolute value 1 , for by the extremal property of $f$, we must have such equality at the point $z_{n}$. q.e.d.

As a consequence of this lemma, the result of Carleson on interpolation sets in the unit disc which we quoted in the introduction can be reformulated in the following way.

THEOREM 5.2. The set $S=\left\{z_{n}\right\}$ of points in the open unit disc is an interpolation set for $U$ if and only if there is $\delta>0$ such that $d_{U}\left(z_{n} ; S\right) \geqq \delta$ holds for all $n$.

We next consider a problem which is of intrinsic interest and which, in addition, will be of importance for our later work. Suppose $V$ to be an open subset of $U$ and $\left\{z_{n}\right\}$ to be a set of points in $V$. What conditions on $V$ and the set $\left\{z_{n}\right\}$ guarantee that if $\left\{z_{n}\right\}$ is an interpolation set for $V$, then it is also one for $U$ ? Our next theorem provides a partial answer.

THEOREM 5.3. Let $V$ be a simply connected subset of $U$ such that $U \backslash V$ is also connected. Let $\partial V$ consist of the arc $\lambda$ with end points $\alpha$ and $\beta$, lying in the unit circle, and the continuum $K$ which lies in $U$ except for its two points $\alpha$ and $\beta$. Let $S=\left\{z_{n}\right\}$ be a set of points of $V$ which clusters only on the interior of $\lambda$. Under these conditions, if $\left\{z_{n}\right\}$ is an interpolation set for $V$, it is also one for $U$.

Proof. By the invariant formulation of Carleson's result, we have that

$$
\inf _{n} d_{V}\left(z_{n} ; S\right) \geqq \delta>0 .
$$

From this, we shall conclude that for some $\delta_{1}$

$$
\inf _{n} d_{U}\left(z_{n} ; S\right) \geqq \delta_{1}>0 .
$$

We start the argument by proving that $\sum_{n}\left(1-\left|z_{n}\right|\right)$ converges. Since $V$ is simply connected, there exists a conformal map $F$ of $U$ onto $V$. Define $z_{n}^{\prime}$ by the condition that $z_{n}=F\left(z_{n}^{\prime}\right)$. The set $\left\{z_{n}\right\}$ is then an interpolation set for $U$, and consequently, we have $\Sigma_{n}\left(1-\left|z_{n}^{\prime}\right|\right)<\infty$. The function $\boldsymbol{F}$ can be continued across the arc $\lambda_{1}=F^{-1}[\lambda]$, and the points $z_{n}^{\prime}$ then cluster only in the interior of $\lambda_{1}$. Let $M$ be a convex, closed set containing the set $\left\{z_{n}^{\prime}\right\}$ and lying in the domain of holomorphy of $F$. On $M,\left|F^{\prime}\right|$ will be bounded, say by $C$. We can choose $M$ so that for all but finitely many $n$, the point $z_{n}^{\prime}|| z_{n}^{\prime} \mid$ lies in $M$. Then for those $n$ for which $z_{n}^{\prime} /\left|z_{n}^{\prime}\right| \in M$, we have the estimate 


$$
\begin{aligned}
1-\left|z_{n}\right| & =\left|F\left(\frac{z_{n}^{\prime}}{\left|z_{n}^{\prime}\right|}\right)\right|-\left|F\left(z_{n}^{\prime}\right)\right| \leqq\left|F\left(\frac{z_{n}^{\prime}}{\left|z_{n}^{\prime}\right|}\right)-F\left(z_{n}^{\prime}\right)\right| \\
& =\left|\int F^{\prime}(z) d z\right| \leqq C\left|\frac{z_{n}^{\prime}}{\left|z_{n}^{\prime}\right|}-z_{n}^{\prime}\right|=C\left(1-\left|z_{n}^{\prime}\right|\right)
\end{aligned}
$$

where the integral is over the interval $\left[z_{n}^{\prime}, z_{n}^{\prime}|| z_{n}^{\prime} \mid\right]$. But since $\Sigma\left(1-\left|z_{n}^{\prime}\right|\right)<\infty$, it follows that $\Sigma\left(1-\left|z_{n}\right|\right)<\infty$.

Since $\Sigma\left(1-\left|z_{n}\right|<\infty\right.$, the Blaschke product $B$ associated with the set $\left\{z_{n}\right\}$ converges in $U$. By hypothesis the set $\left\{z_{n}\right\}$ does not cluster on $K$, and so the function $B$ is bounded away from zero on $K$, say $|B(z)| \geqq \eta$ for $z \in K$. For $n=1,2,3, \cdots$, let $B_{n}$ be the Blaschke product given by (5.1).

Fix an $n$, and choose, by virtue of a normal families argument, an $f_{n}$ such that $\left\|f_{n}\right\|_{V} \leqq 1$ and $\left|f_{n}\left(z_{k}\right)\right|=0$ for $z_{k} \in S, z_{k} \neq z_{n}$, and such that $\left|f_{n}\left(z_{n}\right)\right|=d_{V}\left(z_{n} ; S\right)$. We consider the quotient $f_{n}\left(z_{n}\right) / B_{n}\left(z_{n}\right)$. We have

$$
\left|\frac{f_{n}\left(z_{n}\right)}{B_{n}\left(z_{n}\right)}\right|=\lim _{N}\left|\frac{f_{n}\left(z_{n}\right)}{B_{n}^{(N)}\left(z_{n}\right)}\right|
$$

where $B_{n}^{(N)}$ is the $N$ th partial product of $B_{n}$. For fixed $N$, we have, by virtue of the maximum modulus theorem, the estimate

$$
\left|\frac{f_{n}\left(z_{n}\right)}{B\left(\begin{array}{l}
N \\
n
\end{array}\left(z_{n}\right)\right.}\right| \leqq \frac{\left\|f_{n}\right\|_{V}}{\inf \left\{\left|B\left(\begin{array}{l}
N \\
n
\end{array}\right)(z)\right|: z \in V\right\}} .
$$

We have $\left\|f_{n}\right\|_{V}=1$, and since $\left|B_{n}^{(N)}(z)\right|=1$ for all $z$ in $\lambda$, it follows that

$$
\begin{aligned}
\inf \left\{\left|B_{n}^{(N)}(z)\right|: z \in \partial V\right\} & =\inf \left\{\left|B_{n}^{(N)}(z)\right|: z \in K\right\} \\
& \geqq \inf \{|B(z)|: z \in K\}
\end{aligned}
$$

whence

$$
\left|\frac{f_{n}\left(z_{n}\right)}{B_{n}\left(z_{n}\right)}\right| \leqq \frac{1}{\eta} .
$$

From (5.2) and (5.4) together with the definition of $f_{n}$, we may conclude that $\left|B_{n}\left(z_{n}\right)\right| \geqq \delta \eta$. Thus $S$ is an interpolation set for $U$, and we have the desired result. We can now prove a theorem in the annular case.

THEOREM 5.4. Let $\left\{z_{n}\right\}$ be a set of points in the annulus $A=\{z ; r<|z|<1\}$. If $\left\{z_{n}\right\}$ clusters only on $\Lambda$, then $\left\{z_{n}\right\}$ is an interpolation set for $A$ if and only if it is an interpolation set for $U$.

Proof. Suppose that $\left\{z_{n}\right\}$ is an interpolation set for $A$ which clusters only on $\Lambda$. Again, we commence by proving that $\Sigma\left(1-\left|z_{n}\right|\right)<\infty$. We write 


$$
\left\{z_{n}\right\}=\left\{z_{n}\right\}_{n \in N_{1}} \cup\left\{z_{n}\right\}_{n \in N_{2}}
$$

where $n \in N_{1}$ if and only if $\operatorname{Re} z_{n} \geqq 0$ and $n \in N_{2}$ if and only if $\operatorname{Re} z_{n}<0$. Let $V$ be given by

$$
V=\{z \in A: \arg z \in(-\pi+\varepsilon, \pi-\varepsilon)\}
$$

where $\varepsilon$ is small and positive. Then $\left\{z_{n}\right\}$ is an interpolation set for $A$, and consequently $\left\{z_{n}\right\}_{n \in N_{1}}$ is one for $V$. The preceding theorem applies to $V$ and the set $\left\{z_{n}\right\}_{n \in N_{1}}$ and so it follows that $\Sigma_{n \in N_{1}}\left(1-\left|z_{n}\right|\right)<\infty$. Similarly, we have that $\sum_{n \in N_{2}}\left(1-\left|z_{n}\right|\right)$ is finite. Thus, the Blaschke product $B$ associated with the unit disc and the set $\left\{z_{n}\right\}$ converges. For $n=1,2,3, \cdots$, let $B_{n}$ be given by (5.1). Because $\left\{z_{n}\right\}$ is an interpolation set for $A$, there is a constant $M$ such that for each $n$, there is a function $f_{n} \in H_{\infty}[A]$ with the property that $\left\|f_{n}\right\|_{A} \leqq M, f_{n}\left(z_{k}\right)=0$ for $k \neq n$, and $f_{n}\left(z_{n}\right)=1$. (This uniformity depends on the open mapping theorem of Banach space theory. For a discussion, see $\left[12\right.$, p. 196].) Since $\left\{z_{n}\right\}$ does not cluster on $\{z:|z|=r\}$, there is an $\eta>0$ such that $\left|B\left(r e^{i \theta}\right)\right| \geqq \eta$ holds uniformly in $\theta$. Arguing essentially as in the preceding proof, we find that

$$
\left|\frac{f_{n}\left(z_{n}\right)}{B_{n}\left(z_{n}\right)}\right| \leqq \frac{M}{\eta}
$$

holds for all $n$. Thus $\left|B_{n}\left(z_{n}\right)\right| \geqq \eta / M$. This estimate is uniform in $n$, and so the set $\left\{z_{n}\right\}$ is an interpolation set for $U$.

That an interpolation set for $U$ which lies in $A$ is an interpolation set for $A$ is clear, and so we have our theorem.

This theorem admits the expected formulation in terms of the function $d_{A}$ as is shown by the following corollary.

Corollary 5.5. A set $S=\left\{z_{n}\right\}$ of points of $A$ which clusters only on $\Lambda$ is an interpolation set for $A$ if and only if there is $\delta>0$ such that $\inf _{n} d_{A}\left(z_{n} ; S\right) \geqq \delta$.

Proof. The necessity is quite clear: The preceding theorem shows that if $S$ is an interpolation set for $A$, then it is one for $U$, and so by Theorem 5.2,

$$
d_{U}\left(z_{n} ; S\right) \geqq \delta>0
$$

for some $\delta$ and all $n$. But $d_{A}\left(z_{n} ; S\right) \geqq d_{U}\left(z_{n} ; S\right)$.

Conversely, the statement that $d_{A}\left(z_{n} ; S\right) \geqq \delta>0$ for all $n$ implies the existence of $M$ such that there is, for all $n$, an $f_{n}$ with $\left\|f_{n}\right\|_{A} \leqq M$ and $f_{n}\left(z_{n}\right)=1$ while $f_{n}\left(z_{k}\right)=0$ for $k \neq n$. But then the last proof shows that if $B_{n}$ is as in that proof, the numbers $\left|B_{n}\left(z_{n}\right)\right|$ are uniformly bounded away from zero. Thus $S$ is an interpolation set for $U$, and the result follows.

We now pass to a more general setting, that of a finite open Riemann surface $R$ which is embedded in the compact surface $R_{0}$ as indicated in the introduction. Let $S$ be a set in $R$. 
Let $\Gamma_{j}^{\prime}$ be an analytic simple closed curve in $R$ such that $\Gamma_{j}$ and $\Gamma_{j}^{\prime}$ together constitute the boundary of an annulus $A_{j}$ in $R$. We may assume that $\bar{A}_{j} \cap \bar{A}_{k}=\varnothing$ for all $j \neq k$. We set $S_{j}=S \cap A_{j}$, and we can then assume that $S=\bigcup_{j=1}^{m} S_{j}$.

THEOREM 5.6. If the set $S$ clusters only on $\partial R$, it is an interpolation set for $R$ if and only if $S_{j}$ is an interpolation set for the annulus $A_{j}$ for each $j$.

The proof of Theorem 5.6 will depend on a pair of lemmas.

LEMMA 5.7. If there is a nonconstant function $f \in H_{\infty}\left[A_{1}\right]$ which vanishes on $S_{1}$, then there is an $F_{1} \in H_{\infty}[R]$ whose zero set includes $S_{1}$ and which is bounded away from zero on the set $S \backslash S_{1}$.

Proof. Let $R_{1}$ be the Riemann surface $R_{0} \backslash \Delta_{1}$. The result of Ahlfors cited in Theorem 3.1 guarantees the existence of a holomorphic function $\Phi_{1}$ mapping $R_{1}$ onto $U$ which takes $\Gamma_{1}$ continuously onto $\Lambda=\partial U$. The function $\Phi_{1}$ is $r$-to-one from $\Gamma_{1}$ onto $\Lambda$ for some $r \geqq 1$.

Choose an analytic simple closed curve $\Gamma_{1}^{\prime \prime}$ in $A_{1}$ which, together with $\Gamma_{1}$ bounds an annulus $A_{1}^{\prime \prime}$ such that on $\overline{A_{1}^{\prime \prime}}, \Phi_{1}$ is precisely $r$-to-one. We choose $\Gamma_{1}^{\prime \prime}$ so that $A_{1}^{\prime \prime}$ is mapped onto the annulus $\{z: a<|z|<1\}$ for some $a>0$. There is a finite cyclic group $G$ of analytic transformations of $A_{1}^{\prime \prime}$ onto itself under which $\Phi_{1} \mid A_{1}^{\prime \prime}$ is invariant.

The question of the group $G$ is, perhaps not quite trivial. We can make the point explicit as follows: We have that $\Phi_{1}$ maps $A_{1}^{\prime \prime}$ onto $\{z: a<|z|<1\}$ in an $r$-to-one fashion. Now $A_{1}^{\prime \prime}$ is an annulus, and so there are $b>0$ and $\Psi$ such that $\Psi$ maps $\{z: b<|z|<1\}$ conformally and one-to-one onto $A_{1}^{\prime \prime}$. The composition $\Phi_{1} \circ \Psi$ maps $\{z: b<|z|<1\}$ in an $r$-to-one fashion onto $\{z: a<|z|<1\}$. We assert that $\Phi_{1}(\Psi(z))=z^{r}$ or $z^{-r}$. This is so, for by the reflection principle, $\Phi_{1} \circ \Psi$ can be continued to a function holomorphic in the closed plane except for one of the points 0 and $\infty$, one of which must be a pole, the other a zero. Thus $\Phi_{1} \circ \Psi$ is rational. But now it follows easily that $\Phi_{1}(\Psi(z))=z^{r}$ or $z^{-r}$. The map $\Phi_{1} \circ \Psi$ is thus invariant under the cyclic group of transformations generated by the transformation $z \rightarrow z \exp (2 \pi i / r)$ and we have our group $G$.

The set $S_{1}$ clusters only on $\Gamma_{1}$, and so the set $S_{1}^{\prime \prime}=S \cap A_{1}^{\prime \prime}$ contains all but finitely many of the points of the set $S_{1}$. The function $f$ then vanishes on the set $S_{1}^{\prime \prime}$.

Define $f_{1}$ by $f_{1}(z)=\prod_{g \in G} f(g(z))$. The function $f_{1}$ is nonconstant and invariant under $G$. This being so, we may regard it as a function on the annulus $\{z: a<|z|<1\}$; so regarded, it vanishes on the set $\Phi_{1}\left[S_{1}^{\prime \prime}\right]$.

It follows that there exists a function $f_{2}$ in $H_{\infty}[U]$ whose zero set is the set $\Phi_{1}\left[S_{1}^{\prime \prime}\right]$. Consider $F=f_{2} \circ \Phi_{1}$. This function is nonconstant and vanishes on the set $S_{1}^{\prime \prime}$. Consequently, $F$ can vanish only at only finitely many of the points of $S \backslash S_{1}^{\prime \prime}$. We can construct a function $H$ on $R_{1}$ with the following properties: 
(i) $H$ is bounded near $\Gamma_{1}$;

(ii) $H$ has a zero at each point of $S_{1} \backslash S_{1}^{\prime \prime}$;

(iii) $H$ has a pole at each point of $S \backslash A_{1}$ at which $F$ vanishes. These poles are to be the sole singularities of $H$, and their multiplicity is to be that of the zero of $F$ at the given point.

The function $F_{1}$ given by $F_{1}(z)=F(z) H(z)$ is a function in $H_{\infty}[R]$ which has the desired properties.

Lemma 5.8. If the set $S_{1}$ is an interpolation set for $A_{1}$ which clusters only on $\Gamma_{1}$, then $S_{1}$ is an interpolation set for $R$.

Proof. The proof of this lemma depends on a theorem of Hoffman [12, p. 205] according to which a set $E$ in the surface $R$ is an interpolation set for $R$ if and only if the following conditions are met: (1) $E$ is discrete in its relative topology as a subset of $\mathfrak{M}[R]$; (2) $\bar{E}$, the closure of $E$ in $\mathfrak{M}[R]$, is homeomorphic to the Cech compactification of $E$; (3) $\bar{E}$ is a hull in $\mathfrak{M}[R]$ in the sense that given $q \notin \bar{E}$, there is $f \in H_{\infty}[R]$ such that $\hat{f}(q) \neq 0$ but $\hat{f}(p)=0$ for every $p \in E$.

We shall prove the lemma by showing that the set $S_{1}$ in $R$ has the indicated properties. To begin with, it is clear that $S_{1}$ is discrete in its relative topology as a subset of $\mathfrak{M}[R]$.

Since $A_{1} \subset R$, we have a natural inclusion $\kappa: H_{\infty}[R] \rightarrow H_{\infty}\left[A_{1}\right]$, and this induces the adjoint $\kappa^{*}: \mathfrak{M}\left[A_{1}\right] \rightarrow \mathfrak{M}[R]$. We have seen in the proof of Theorem 2.6 that if $\phi \in \mathfrak{M}[R]$, and if $\phi$ lies in a fiber over a point of $A_{1}$ or $\Gamma_{1}$, then $\phi$ admits a unique extension to an element of $\mathfrak{M}\left[A_{1}\right]$. This implies that $\kappa^{*}$ acts as a homeomorphism on $S_{1} \subset \mathfrak{M}\left[A_{1}\right]$ and so, since $S_{1}$ is an interpolation set for $A_{1}$, that $S_{1} \subset \mathfrak{M}[R]$ is topologically $\beta\left(S_{1}\right)$.

Our proof concludes by showing that the hull of $S_{1}$ in $\mathfrak{M}[R]$ is the set $S_{1}$. We must show that if $\phi_{0} \in \mathfrak{M}[R] \backslash S_{1}$, then there is $f \in H_{\infty}[R]$ such that $\hat{f}\left(\phi_{0}\right) \neq 0$ but $\hat{f}(z)=0$ for all $z \in \bar{S}_{1}$.

Let $\phi_{0}$ lie in the fiber $\mathfrak{M}_{\alpha}[R]$ over the point $\alpha \in \Gamma_{1}$ and assume $\phi_{0}$ is not in $\bar{S}_{1}$. Let $\Phi_{2}$ be a function, existing by the theorem of Ahlfors, on $R$ which maps $R$ holomorphically onto $U$, which is continuous on $\bar{R}$, and which takes $\partial R$ onto $\Lambda$. We can find a neighborhood, $M$, of $\alpha$ in $\bar{R}$ on which $\Phi_{2}$ is one-to-one. Let $S_{1}{ }^{\prime}$ be a subset of $S_{1}$ such that $S_{1} \backslash S_{1}^{\prime}$ clusters only off some proper closed subarc of $\Gamma_{1}$ contained in $M$ and containing $\alpha$ in its interior. We require also that $S_{1}^{\prime}$ lie in $M$ and cluster only on some proper closed subarc of $\Gamma_{1}$ which is contained in $M$. Then $S_{1}^{\prime}$ is an interpolation set for $M$. It follows by Theorem 5.3 that $\Phi_{2}\left[S_{1}^{\prime}\right]$ is an interpolation set for $U$.

The map $\Phi_{2}$ induces an inclusion $\mathrm{\imath}: H_{\infty}[U] \rightarrow H_{\infty}[R]$, and we have the adjoint $\imath^{*}: \mathfrak{M}[R] \rightarrow \mathfrak{M}[U]$. The point $\phi_{0}$ is taken by $\imath^{*}$ to a point not in $\Phi_{2}\left[S_{1}^{\prime}\right]^{-} \subset \mathfrak{M}[U]$. Since $\Phi_{2}\left[S_{1}^{\prime}\right]$ is an interpolation set for $U$, the theorem of Hoffman provides a function $f_{1} \in H_{\infty}[U]$ such that $f_{1}\left(\Phi_{2}(z)\right)=0$ for $z \in S_{1}^{\prime}$, and $\hat{f}_{1}\left(1^{*} \phi_{0}\right) \neq 0$. The 
function $f_{1} \circ \Phi_{2}$ lies in $H_{\infty}[R]$, and we have that $\left(f_{1} \circ \Phi_{2}\right) \wedge(z)=0$ for $z \in S_{1}^{\prime}$, while $\left(f_{1} \circ \Phi_{2}\right) \wedge\left(\phi_{0}\right) \neq 0$.

We now observe that there is a function $f_{2} \in H_{\infty}[R]$ such that $f_{2}(z)=0$ if $z \in S_{1} \mid S_{1}^{\prime}$ and such that $f_{2}$ is bounded away from zero near $\alpha$. To obtain such a function, we argue as follows. Let $N$ be a small disc in $R_{0}$ about $\alpha$, and consider the annulus $A_{1} \cup N$. By mapping this annulus into the plane and invoking Theorems 5.4 and 5.3, we find that $S_{1} \backslash S_{1}^{\prime}$ is an interpolation set for $A_{1} \cup N$. Next we use Lemma 5.7 to find a function $g$ holomorphic on the surface $R \cup N$ which vanishes on $S_{1} \backslash S_{1}^{\prime}$. (Note that although the Lemma 5.7 was proved for the case of a domain with analytic boundary, it is applicable in the present case, since by an analytic homeomorphism the domain $R \cup N$ can be mapped into a domain with analytic boundary. For a discussion of such mappings, see $\S 8$ below. We note that this section is independent of the rest of the paper.)

If $g(\alpha) \neq 0$, we let $f_{2}=g$. If, on the other hand, $g(\alpha)=0$, we can find a function $g_{1}$ holomorphic on $R \cup N$ which has a single zero, this zero located at $\alpha$. Furthermore, the order of the zero of $g_{1}$ at $\alpha$ can be made to coincide with the order of the zero of $g$ at $\alpha$. Since we can choose $g_{1}$ to be bounded away from zero on $R \cup N$ except in a neighborhood of $\alpha$, the function $g / g_{1}$ has the properties desired of $f_{2}$.

The function $f_{2}\left(f_{1} \circ \Phi_{2}\right)$ is in $H_{\infty}[R]$, and it vanishes on the set $S_{1}$, but $\left(f_{2}\left(f_{1} \circ \Phi_{2}\right)\right) \wedge$ does not annihilate $\phi_{0}$.

So far we have assumed $\alpha_{0} \in \Gamma_{1}$; the contrary case is trivial. It follows that the hull of the set $S_{1}$ in $\mathfrak{M}[R]$ is just $\bar{S}_{1}$, and we have our lemma.

With these two lemmas, the thorem can be established with no serious difficulty.

Proof of Theorem 5.6. Plainly if $S$ is an interpolation set for $R$, then $S_{j}$ must be an interpolation set for the annulus $A_{j}$ for $j=1,2,3, \cdots, m$.

Conversely, suppose that $S_{j}$ is an interpolation set for $A_{j}$ for each $j$. Apply Lemma 5.7 to find functions $F_{1}, \cdots, F_{m}$ in $H_{\infty}[R]$ which satisfy $F_{j}(z)=0$ if $z \in S_{j}$ and which are bounded away from zero on $S \backslash A_{j}$. Set $G_{i}=F_{1} \cdots F_{i-1} F_{i+1} \cdots F_{m}$. Then we have that $G_{i}$ is bounded away from zero on $S_{i}$ and that $G_{i}$ is identically zero on the set $S_{j}$ for $i \neq j$.

Let $\alpha$ be a bounded complex valued function on $S$. Lemma 5.8 yields functions $H_{1}, \cdots, H_{m}$ in $H_{\infty}[R]$ such that $H_{j}(z)=\alpha(z) / G_{j}(z)$ for $z \in S_{j}$. If we set $L(z)$ $=\sum_{j=1}^{m} G_{j}(z) H_{j}(z)$, then $L(z)=\alpha(z)$ for all $z \in S$, and we have our theorem.

We can use the result just obtained to give a conformally invariant, intrinsic characterization of interpolation sets in $R$.

THEOREM 5.9. Let $R$ be a finite open Riemann surface, and let $S=\left\{z_{n}\right\}$ be a set of points in $R$. Then $S$ is an interpolation set for $R$ if and only if there is $\delta>0$ such that 


$$
\delta \leqq \inf _{n} d_{R}\left(z_{n} ; S\right) .
$$

Proof. The necessity of the condition is evident by virtue of the open mapping theorem as in the proof of Theorem 5.4.

The sufficiency is also immediate by virtue of the results we have just obtained. Suppose (5.6) satisfied. Thus, if $z_{n} \in A_{j}$, we have

$$
\begin{aligned}
\delta & \leqq d_{R}\left(z_{n} ; S\right) \\
& =\sup \left\{\left|f\left(z_{n}\right)\right|: f \in H_{\infty}[R], f(z)=0 \quad \text { for } z \in S \backslash\left\{z_{n}\right\}, \text { and }\|f\|_{R} \leqq 1\right\} \\
& \leqq \sup \left\{\left|f(z)_{n}\right|: f \in H_{\infty}[R], f(z)=0 \quad \text { for } z \in S_{j} \backslash\left\{z_{n}\right\},\|f\|_{R} \leqq 1\right\} \\
& \leqq d_{A_{j}}\left(a_{n} ; S_{n}\right) .
\end{aligned}
$$

These estimates are uniform in $n$ for $z_{n} \in A_{j}$, and so by Theorem 5.5, the set $S_{j}$ is an interpolation sert fo $A_{\jmath}$. The desired conclusion now follows from Theorem 5.6.

6. Interpolation and uniformization. We now come to a pair of theorems which relate interpolation sets in a finite Riemann surface to interpolation sets in its universal covering surface.

We continue to work on the finite Riemann surface $R$ embedded in the compact surface $R_{0}$ as indicated in the introduction. We let $F: U \rightarrow R$ be a uniformizing map whose associated group of covering transformations we denote by $G$. The group $G$ is isomorphic to the fundamental group of $R$, and so it is finitely generated.

We shall need to make use of certain information about the fundamental polygon of $G$, but before proceeding to this, it is convenient to introduce two notations. If $z, w \in U$, we set $[z, w]=|(z-w) /(1-z \bar{w})|$, and we set $\rho(z, w)$ $=\frac{1}{2} \log (1+[z, w]) /(1-[z, w])$. Thus, $\rho$ is the usual hyperbolic metric in the unit disc.

We shall denote by $D$ the "Poincaré normal fundamental polygon" of the group $G$, i.e., $D=\{z \in U: \rho(z, 0) \leqq \rho(z, \gamma(z))$ for all $\gamma \in G\}$. The set $D$ is known to consist of an open set in $U$ together with the part of its boundary which lies in $U$. This boundary consists of arcs of hyperbolic straight lines, i.e., of arcs of circles orthogonal to the unit circle together with certain arcs in the unit circle. We shall speak of a maximal hyperbolic line segment or of a maximal arc of the unit circle contained in $\partial D$ as a side of $D$. A recent theorem of Heins [11] assures us that since $G$ is finitely generated, $D$ has only a finite number of sides. (Another proof of this fact is due to L. Greenberg, but it remains unpublished as yet.)

The domain $D$ has the property that on its interior, the map $F$ is one-to-one. Furthermore, $F$ maps $D$ onto $R$. The function $F$ is actually analytic in a neighborhood of $\bar{D}$, and it carries the sides of $D$ which lie in $\Lambda$ onto the curves constituting the boundary of $R$ in $R_{0}$. One can show that the group $G$ identifies by 
pairs the sides of $D$ lying in $U$. If $z \in U$, then either there is a unique $\gamma \in G$ such that $\gamma(z)$ lies in the interior of $D$, or else there are certain of the sides of $D$ lying in $U$ each of which contains a point which is the image of $z$ under some element of $G$. Since $D$ has only finitely many sides, we see that for only finitely many $\gamma \in G$ is it the case that $\overline{\gamma[D]} \cap \bar{D} \neq \varnothing$.

The first question we shall consider is the following: Suppose given a set $S$ in $U$. Under what conditions is $F[S]$ an interpolation set for $R$ ? An answer is as follows.

THEOREM 6.1. Let $S \subset U$ and let $S$ be invariant under the group $G$ in the sense that $\gamma[S]=S$ for all $\gamma \in G$. If $S$ is an interpolation set for $U$, then $F[S]$ is an interpolation set for $R$.

Before giving the proof of the theorem, we point out a corollary.

COROLlaRY 6.2. Let $S$ be a set in $U$ which is invariant under the group $G$, and let $\alpha$ be a bounded, complex valued function on $S$ which is also invariant under $G$. If $S$ is an interpolation set for $U$, then there is a function $f \in H_{\infty}[U]$ which is invariant under the group $G$ and which satisfies $f(s)=\alpha(s)$ for all $s \in S$.

Proof of Theorem 6.1. The domain $D$ has finitely many sides, $m$ of which, say $\lambda_{1}, \cdots, \lambda_{m}$, are arcs in the unit circle. We can choose the indexing so that $F\left[\lambda_{j}\right]=\Gamma_{j}$. We consider $\lambda_{1}$ whose end points we assume to be $\alpha$ and $\beta$. The points $\alpha$ and $\beta$ are identified by some element, say $\gamma_{0}$, of the group $G$. Let $G_{0}$ be the subgroup of $G$ generated by $\gamma_{0}$.

Let $L_{\alpha}$ and $L_{\beta}$ be the components of the boundary of $D$ lying in $U$ and containing $\alpha$ and $\beta$ respectively. Let $\alpha^{\prime}$ and $\beta^{\prime}$ lie in $L_{\alpha}$ and $L_{\beta}$ near $\alpha$ and $\beta$ respectively, and let $\alpha^{\prime}$ and $\beta^{\prime}$ be identified by $\gamma_{0}$. Let $\lambda_{1}^{\prime}$ be an arc in $D$ which connects $\alpha^{\prime}$ and $\beta^{\prime}$. Finally let $\Delta$ be the subset of $D$ bounded by $\lambda_{1}, \lambda_{1}$, and the arcs $\alpha \alpha^{\prime}$ and $\beta \beta^{\prime}$ of $L_{\alpha}$ and $L_{\beta}$.

Under $F, \Delta$ is mapped onto a certain annulus, say $A_{1}$, in $R$ with $\Gamma_{1}=F\left[\lambda_{1}\right]$ constituting half the boundary of $A_{1}$. We let $S_{1}$ be the subset of $S$ consisting of those $z \in S$ which lie in $\Delta$, and we shall show that $F\left[S_{1}\right]$ is an interpolation set for $A_{1}$. The theorem is then an immediate consequence of Theorem 5.6.

Let $D_{0}$ be the orbit of $\Delta$ under $G_{0}$. Thus $D_{0}$ is a subset of $U$ which contains $S_{0}=\bigcup\left\{\gamma_{0}^{n}\left[S_{1}\right]: n=0, \pm 1, \pm 2, \pm 3, \cdots\right\}$. The group $G_{0}$ acts as a group of analytic transformations of $D_{0}$ onto itself, and if we let $F_{0}=F \mid D_{0}$, then $F_{0}$ is invariant under $G_{0}$, and it maps $D_{0}$ onto $A_{1}$. By hypothesis, the set $S$ is an interpolation set for $U$, and so a fortiori, the set $S_{0}$ is an interpolation set for $D_{0}$. Let $\alpha$ be a bounded complex valued function on $S_{1}$. There exists $f \in H_{\infty}\left[D_{0}\right]$ such that $f(\gamma(z))=\alpha(z)$ for all $\gamma \in G_{0}$ and all $z \in S_{1}$.

We can now apply the Markov-Kakutani fixed point theorem to obtain a function $f_{0}$ which is invariant under $G_{0}$ and which satisfies $f_{0} \in H_{\infty}\left[D_{0}\right]$ and 
$f(z)=\alpha(z)$ for $z \in S_{1}$. The argument is quite analogous to that used in Theorem 2.1. Let $K$ be the set of all $f \in H_{\infty}\left[D_{0}\right]$ such that $f(\gamma(z))=f(z)$ for all $\gamma \in G_{0}$ and all $z \in S_{1}$ and which, in addition, satisfy $\|f\|_{D_{0}} \leqq C$ for some constant $C$ chosen large enough that $K$ is nonvoid. Then $K$ is a convex set in the space $H\left[D_{0}\right]$ of all functions holomorphic in $D_{0}$. Furthermore, $K$ is compact in the topology of uniform convergence on compact subsets of $D_{0}$. Define operators $\left\{\Phi_{\gamma}\right\}_{\gamma \in G_{0}}$ by means of $\left(\Phi_{\gamma} f\right)(z)=f(\gamma(z))$ for all $z \in D_{0}$ and all $f \in H_{\infty}\left[D_{0}\right]$. As in Theorem 2.1, there is $f_{0} \in K$ which is a fixed point for the group $\left\{\Phi_{\gamma}\right\}_{\gamma \in G_{0}}$.

The function $f_{0}$ is invariant under $G_{0}$, and hence $f_{0} \circ F_{0}^{-1}$ is a well defined bounded function on $A_{1}$. It takes the value $\alpha(z)$ at the point $F(z)$ for all $z \in S_{1}$. We may conclude that $F\left[S_{1}\right]=F\left[S_{0}\right]$ is an interpolation set for $A_{1}$, and the theorem follows.

The preceding theorem admits a converse which can be formulated as follows. Let $F$ be as above.

THEOREM 6.3. Let $S \subset R$, and suppose $S$ to be an interpolation set for $R$. Then the set $F^{-1}[S]$ is an interpolation set for $U$.

Proof. Let $S=\left\{\zeta_{k}\right\}_{k=1}^{\infty}$, and determine $z_{k} \in D$ by $F\left(z_{k}\right)=\zeta_{k}$. Since $S$ is an interpolation set, we may conclude that $\sum_{\gamma \in G, k=1,2, \ldots}\left(1-\left|\gamma\left(z_{k}\right)\right|\right)<\infty$ and, by Theorem 5.9, that for some $\delta>0$ we have for all $n$

$$
\delta \leqq \sup \left\{\left|\left(z_{n}\right)\right|: f \in H_{\infty}[U], \quad\|f\|_{U} \leqq 1, f\left(\gamma\left(z_{k}\right)\right)=0\right.
$$

for $k \neq n$ and all $\gamma \in G\}$. This latter estimate is uniform in $n$.

We have noted that for only finitely many elements $\gamma$ of $G$, say $e, \gamma_{1}, \cdots, \gamma_{m}$, is the case that $\bar{D} \cap \overline{\gamma[\bar{D}]} \neq \varnothing$. Note that, as usual, $e$ is the identity of $G$.

Consider the Blaschke product $B_{n}$ given by

$$
B_{n}(z)=\prod \frac{\gamma\left(z_{n}\right)}{\left|\gamma\left(z_{n}\right)\right|} \frac{z-\gamma\left(z_{n}\right)}{1-\overline{\gamma\left(z_{n}\right)} z}
$$

where the product is extended over all $\gamma \in G$ such that $\gamma \neq e, \gamma_{1}, \cdots, \gamma_{m}$. We say that the functions $B_{n}$ are bounded away from zero on $\bar{D}$, the bound being independent of $n$. This is so, for if $B$ is the Blaschke product whose zeros are the points $\gamma\left(z_{k}\right)$ for $k=1,2, \cdots$ and $\gamma \neq e, \gamma_{1}, \cdots, \gamma_{m}$, then the function $B$ is holomorphic in $U$ and zero free on $\bar{D}$. For every $n$, the zeros of $B$ include those of $B_{n}$, and so we have uniformly in $n$ and $z$ for $z \in \bar{D}, 0<|B(z)| \leqq\left|B_{n}(z)\right|$. Hence, the functions $\left|B_{n}\right|$ are bounded away from zero on $\bar{D}$, their common lower bound not depending on $n$. Let this common lower bound be $\varepsilon$.

Define now a function $\phi$ on $\bar{D}$ by means of $\phi(z)=\prod_{k=1}^{m}\left|\left(z-\gamma_{k}(z)\right) /\left(1-\overline{\gamma_{k}(z)} z\right)\right|$. We shall show that $\phi$ has a strictly positive lower bound on $\bar{D}$. We have that for $z \in \bar{U},\left|1-\overline{\gamma_{k}(z)} z\right| \leqq 2$ so that $\phi(z) \geqq 2^{-m} \prod_{k=1}^{m}\left|z-\overline{\gamma_{k}(z)}\right|$. The functions 
$z-\gamma_{k}(z)$ are continuous on $\bar{U}$, and since each element of $G$ other than the identity is free of fixed points in $U$, they do not vanish in $U$. If we can show that $\gamma_{k}$ has no fixed point on $\partial D$, we shall have the desired result concerning $\phi$. Now each $\gamma_{k}$ has at most two fixed points in $\bar{U}$. Since for each $k, \overline{\gamma_{k}[D]} \cap \bar{D} \subset \partial D$, it follows that any fixed point of $\gamma_{k}$ must be one of the end points of one of the arcs $\lambda_{j}$ which constitute the portion of $\partial D$ which lies in $\Lambda$. On the other hand, it is clear that such a point is not a fixed point for any of the $\gamma_{k}$. Thus, we have that there is $\eta$ such that if $z \in D$, then $0<\eta<\phi(z)$ for all $z \in \bar{D}$.

Consider now one of the $z_{n}$. There is a function $f_{0}$ such that $\|f\|_{U} \leqq 1$, $\left|f_{0}\left(z_{n}\right)\right| \geqq \delta>0$ and $f\left(\gamma\left(z_{k}\right)\right)=0$ for $k \neq n$ and all $\gamma \in G$ where, as noted at the outset of the proof, $\delta$ is independent of $n$. We set

$$
g(z)=f_{0}(z) B_{n}(z) \prod_{j=1}^{m} \frac{z-\gamma_{j}\left(z_{n}\right)}{1-\overline{\gamma_{j}\left(z_{n}\right) z}} .
$$

We have then that $\|g\|_{U} \leqq 1$, and we also have that

$$
\begin{aligned}
\left|g\left(z_{n}\right)\right| & =\left|f_{0}\left(z_{n}\right)\right|\left|B_{n}\left(z_{n}\right)\right| \phi\left(z_{n}\right) \\
& \geqq \delta \in \eta .
\end{aligned}
$$

Here $\delta, \varepsilon$, and $\eta$, are independent of the choice of $z_{n}$. Furthermore, we have that $g\left(\gamma\left(z_{n}\right)\right)=0$ if $\gamma \neq e$, and $g\left(\gamma\left(z_{k}\right)\right)=0$ for all $k \neq \eta$ and all $\gamma \in G$. Thus $d_{u}\left(z_{n}, F^{-1}[S]\right) \geqq \varepsilon^{\prime}>0$, this estimate being uniform in $n$. But now, since $F^{-1}[S]$ is invariant under $G$, we can conclude that $d_{v}\left(\gamma\left(z_{n}\right), F^{-1}[S]\right)>\varepsilon^{\prime}$ holds for all $\gamma \in G$ and all $z_{n}$. Thus, by Theorem 5.2, $F^{-1}[S]$ is an interpolation set for $U$, q.e.d.

There is one further theorem in this general vein which we shall consider and which is, however, much simpler than the preceding two. We have introduced the notation $[z, w]=|(z-w) /(1-z \bar{w})|$. A direct calculation shows that if $\phi$ is a conformal map of $U$ onto itself, then $[z, w]=[\phi(z), \phi(w)]$ whence

$$
[\phi(z), w]=\left[z, \phi^{-1}(w)\right] .
$$

THEOREM 6.4. Let $G$ be a group of conformal transformations of $U$ onto itself which is of convergence type, i.e., which is such that for some and therefore all $z \in U, \sum_{\gamma \in G} 1-|\gamma(z)|$ converges. If $z_{0} \in U$, the set $\left\{\gamma\left(z_{0}\right)\right\}_{\gamma \in G}$ is an interpolation set for $U$.

Proof. Having fixed $z_{0}$ and $\gamma_{0} \in G$, define $B\left(z ; \gamma_{0}\right)$ by

$$
B\left(z ; \gamma_{0}\right)=\prod_{\gamma \in G ; \gamma \neq \gamma_{0}} \frac{\overline{\gamma\left(z_{0}\right)}}{\left|\gamma\left(z_{0}\right)\right|} \frac{z-\gamma\left(z_{0}\right)}{1-\overline{\gamma\left(z_{0}\right) z}} .
$$

Since $G$ is of convergence type, this product converges in $U . B\left(\cdot ; \gamma_{0}\right)$ is a holo- 
morphic function in $U$ which vanishes at the points $\gamma\left(z_{0}\right), \gamma \neq \gamma_{0}$, and which does not vanish at $\gamma\left(z_{0}\right)$.

Let $\delta_{0}=B\left(\gamma_{0}\left(z_{0}\right) ; \gamma\right)=\prod_{\gamma \in G ; \gamma \neq \gamma_{0}}\left[\gamma_{0}\left(z_{0}\right), \gamma\left(z_{0}\right)\right]$. We have noted that $\left[\gamma_{0}\left(z_{0}\right), \gamma\left(z_{0}\right)\right]=\left[z_{0}, \gamma_{0}^{-1} \gamma\left(z_{0}\right)\right]$, and so $\delta_{0}=B\left(z_{0} ; e\right)$ where $e$ is the identity of $G$. In particular, $\delta_{0}$ is independent of the choice of $\gamma_{0}$. But this fact simply means that $\left\{\gamma\left(z_{0}\right)\right\}_{\gamma \in G}$ satisfies the condition of Carleson and so is an interpolation set for $U$, q.e.d.

7. Another characterization of interpolation sets. We are able to give one final characterization of interpolation sets in the surface $R$. For the case of a plane domain, the result is in [8]. If $\zeta \in R$, let $g(\cdot, \zeta)$ be the Green's function of $R$ with respect to the point $\zeta$. Thus $g(\cdot, \zeta)$ is a function positive and harmonic on $R \backslash\{\zeta\}$, which has boundary values zero on $\partial R$, and which has a logarithmic singularity at the point $\zeta$. The following theorem relates the Green's function to interpolation.

THEOREM 7.1. Let $\left\{\zeta_{n}\right\}_{n \in N}$ be a set of points in $R$. Then $\left\{\zeta_{n}\right\}_{n \in N}$ is an interpolation set for $R$ if and only if there is an $M$ such that

$$
\sum_{n \in N ; n \neq n_{0}} g\left(\zeta_{n}, \zeta_{n_{0}}\right) \leqq M
$$

holds for all $n_{\mathrm{o}} \in N$.

Proof. The structure of our proof is as follows. We shall first derive the necessity of the condition. Next we obtain the sufficiency in the case that $R$ is an annulus. The sufficiency in this special case then allows us to obtain sufficiency in the general case. We shall continue the notation of Theorems 6.1 and 6.3 concerning the map $F$, the group $G$, and the fundamental polygon $D$ of $G$.

For each $n$, let $z_{n}$ be determined by the conditions $z_{n} \in D, F\left(z_{n}\right)=\zeta_{n}$. We have shown that $\left\{\zeta_{n}\right\}$ is an interpolation set for $R$ if and only if the set $\left\{\gamma\left(z_{n}\right)\right\}_{\gamma \in G, n \in N}$ is an interpolation set for $U$.

Invoking the theorem of Carleson in the disc, $\left\{\zeta_{n}\right\}$ is an interpolation set for $R$ if and only if there is $\delta$ such that

$$
0<\delta \leqq \prod\left[\gamma\left(z_{n}\right), \gamma_{0}\left(z_{n_{0}}\right)\right]
$$

holds for all $\left(\gamma_{0}, n_{0}\right) \in G \times N$. The product extends over all $(\gamma, n)$ in $G \times N \backslash\left(\gamma_{0}, n_{0}\right)$. Taking real logarithms, we find the equivalent condition

$$
-\infty<M^{\prime} \leqq \sum \log \left[\gamma\left(z_{n}\right), \gamma_{0}\left(z_{n_{0}}\right)\right]
$$

Now

$$
\begin{aligned}
\sum \log \left[\gamma\left(z_{n}\right), \gamma_{0}\left(z_{n_{0}}\right)\right]= & \sum_{n \neq n_{0}} \sum_{\gamma \in G} \log \left[\gamma\left(z_{n}\right), \gamma_{0}\left(z_{n_{0}}\right)\right] \\
& +\sum_{\gamma \neq \gamma_{0}} \log \left[\gamma\left(z_{n_{0}}\right), \gamma_{0}\left(z_{n_{0}}\right)\right]
\end{aligned}
$$


It is known $[19$, p. 522] that

$$
g\left(F(z), \zeta_{n_{0}}\right)=-\sum_{\gamma \in G} \log \left[\gamma(z), \gamma_{0}\left(z_{n_{0}}\right)\right] .
$$

Remark that the right side of this equality is independent of the choice of $\gamma_{0} \in G$.

Consider the series $\sum_{\gamma \neq \gamma_{0}} \log \left[\gamma\left(z_{n_{0}}\right), \gamma_{0}\left(z_{n_{0}}\right)\right]$. We have that

$$
\left[\gamma\left(z_{n_{0}}\right), \gamma_{0}\left(z_{n_{0}}\right)\right]=\left[\gamma_{0}^{-1} \gamma\left(z_{n_{0}}\right), z_{n_{0}}\right] \text {. }
$$

As $\gamma$ runs through $G \backslash\left\{\gamma_{0}\right\}, \gamma_{u}^{-1} \gamma$ runs through $G \backslash\{e\}$, and so we find that

$$
\sum_{\gamma \neq \gamma_{0}} \log \left[\gamma\left(z_{n_{0}}\right), \gamma_{0}\left(z_{n_{0}}\right)\right]=\sum_{\gamma \neq e} \log \left[\gamma\left(z_{n_{0}}\right), z_{n_{0}}\right] .
$$

This latter sum is independent of $\gamma_{0}$, and so we have established that the set $\left\{\zeta_{n}\right\}_{n \in N}$ is an interpolation set for $R$ if and only if there exists $M$ such that, independently of $n_{0}$,

$$
\sum_{n \neq n_{0}} g\left(\zeta_{n}, \zeta_{n_{0}}\right)-\sum_{\gamma \neq e} \log \left[\gamma\left(z_{n_{0}}\right), z_{n_{0}}\right] \leqq M
$$

Since the second sum is negative, the necessity of the condition (7.1) is established.

In order to establish the sufficiency of the condition (7.1) in the general case, it would be sufficient to prove that the sum $\sum_{\gamma \neq e} \log \left[\gamma\left(z_{n_{0}}\right), z_{n_{0}}\right]$ is bounded independently of the choice of $z_{n_{0}}$ in $D$, for then the boundedness of the sum (7.2) would be equivalent to that of the sum (7.1).

Suppose that $z_{0} \in D$. If $\gamma \in G$, we have that

$$
\left[\gamma\left(z_{0}\right), z_{0}\right] \geqq \inf \{[\gamma(z), z]: z \in D\} .
$$

We shall call this latter quantity $\delta(\gamma)$. Let $e, \gamma_{1}, \cdots, \gamma_{N}$ be the elements of $G$ with the property that $\overline{\gamma_{j}(D)} \cap \bar{D} \neq \varnothing$. We write then

$$
\begin{aligned}
\sum_{\gamma \neq e} \log \left[\gamma\left(z_{n_{0}}\right), z_{n_{0}}\right]= & \sum_{j=1}^{N} \log \left[\gamma_{j}\left(z_{n_{0}}\right), z_{n_{0}}\right] \\
& +\sum_{\gamma \in \widetilde{G}} \log \left[\gamma_{j}\left(z_{n_{0}}\right), z_{n_{0}}\right] \\
\geqq & \sum_{j=1}^{N} \log \left[\gamma_{j}\left(z_{n_{0}}\right), z_{n_{0}}\right]+\log \prod_{\gamma \in \widetilde{G}} \delta(\gamma)
\end{aligned}
$$

where $\tilde{G}=G \backslash\left\{e, \gamma_{1}, \cdots, \gamma_{N}\right\}$.

Since the elements of $G$ are free of fixed points on $\bar{D}$, we have that $\sum_{j=1}^{N} \log \left[\gamma_{j}\left(z_{n_{0}}\right), z_{n_{0}}\right] \geqq C$ for some $C$, this estimate being uniform in $z_{n_{0}}$ for $z_{n_{0}}$ in $D$. We thus have the estimate

$$
\sum_{\gamma \neq e} \log \left[\gamma\left(z_{n_{0}}\right), z_{n_{0}}\right] \geqq C+\log \prod_{\gamma \in \widetilde{G}} \delta(\gamma) .
$$


So far, we have proceeded in full generality, but we now must specialize a bit, for the infinite product in (7.3) does not seem tractable in the general case. In the annular case, however, it can be handled by an elementary geometric argument. It is to this special case that we now turn our attention.

In the case that $R$ is taken to be an annulus, $A$, the associated group $G$ is infinite cyclic with, say, $\gamma_{0}$ as generator. We can normalize $F$ and $G$ to the extent that each element of $G$ leaves fixed the real diameter of the disc. With these normalizations, $\gamma_{0}$ can be chosen to move points on the real axis to the left. The fundamental domain $D$ is then a domain symmetric with respect to the imaginary axis and bounded by a pair of circular arcs orthogonal to the unit circle and orthogonal to the real axis.

We have to compute $\delta\left(\gamma_{0}^{n}\right)$ for $n= \pm 2, \pm 3, \cdots$. It is evidently enough to consider the case of positive $n$ only. Recall that if $z, w \in U$, then

$$
\rho(z, w)=\frac{1}{2} \log \frac{1+[z, w]}{1-[z, w]}
$$

so that $\rho$ is the usual hyperbolic metric in the disc and is, in particular, additive along hyperbolic straight lines.

Denote by $q$ the point of intersection of the real axis and $\partial D$ which lies in the left half plane. We have then that

$$
\inf \left\{\rho(z, w): z \in D, w \in \gamma_{0}^{n}[D]\right\} \leqq \rho\left(q, \gamma_{0}^{n}(q)\right) .
$$

An elementary geometric argument will allow us to conclude that equality actually holds. We see by a conformal map of the disc onto itself that the assertion of equality in (7.4) is equivalent to the assertion that if $L$ is a hyperbolic straight line in the left half plane which is orthogonal to the real axis and which meets the real axis at $x_{0}$, then the point $x_{0}$ is the point of $L$ nearest, in terms of the metric $\rho$, to the imaginary axis.

Suppose then that $L$ and $x_{0}$ are as given, and let $\rho_{0}=\rho\left(0, x_{0}\right)$. The hyperbolic circle $\left\{z: \rho(z, 0)=\rho_{0}\right\}$ is also a Euclidean circle, and so, by convexity, it meets $L$ in only one point, viz., $x_{0}$. Suppose then that $i y$ is a point on the imaginary axis, and let $c$ be the hyperbolic circle centered at $i y$ and having radius $\rho_{0}$. The circle $c$ is also a Euclidean circle, but its Euclidean center is not the point $i y$ unless $y=0$. However, symmetry shows that the Euclidean center must be on the imaginary axis. The Euclidean radius of $c$ is not greater than $\left|x_{0}\right|$, and so we conclude by convexity that unless $y=0$, the circle $c$ misses $L$.

The conclusion of this argument is that in (7.4) we have equality. Now each of the elements of $G$ is a $\rho$-isometry, and so we have that

$$
\inf \left\{\rho(z, w): z \in D, w \in \gamma_{0}^{n}[D]\right\}=\rho\left(q, \gamma_{0}^{(n-1)}(q)\right)=(n-1) m
$$

where we write $m=\rho\left(q, \gamma_{0}(q)\right)$. This last equality is dependent upon the fact 
that the elements of $G$ leave the real diameter fixed and the fact that $\rho$ is additive along this diameter. Hence we find that $\delta\left(\gamma_{0}^{n}\right) \geqq \tanh ((n-1) m)$ and so $\prod \delta\left(\gamma_{0}^{n}\right) \geqq \prod_{n=1}^{\infty} \tanh ^{2} n m$ where the first product extends over $n= \pm 2, \pm 3, \cdots$. But one easily sees that $\prod_{n=1}^{\infty} \tanh n m$ converges. Thus the bound in (7.3) is uniform in $n$, and our theorem is established in the annular case.

Now suppose that the set $\left\{\zeta_{n}\right\}_{n \in N}$ of points of the suface $R$ satisfies the condition (7.1). For $j=1,2, \cdots, n$, let $\Gamma_{j}^{\prime}$ be a simple closed curve in $R$ which bounds, with $\Gamma_{j}$, an annulus $A_{j}$. Suppose that $A_{1} \cup \cdots \cup A_{n} \supset\left\{\zeta_{n}\right\}_{n \in N}$. Fix upon a $\zeta_{n_{0}}$ in $A_{1}$. By virtue of (7.1), we have that $\sum g\left(\zeta_{n}, \zeta_{n_{0}}\right) \leqq M$, where the sum goes over all $n \in N$ such that $n \neq n_{0}$ and such that $\zeta_{n} \in A_{1}$. Let $g_{A_{1}}\left(\cdot, \zeta_{n_{0}}\right)$ be the Green's function for the annulus $A_{1}$ with singularity $\zeta_{n_{0}}$. On $\Gamma_{1}^{\prime}$ we have

$$
g_{A_{1}}\left(\cdot, \zeta_{n_{0}}\right)<g\left(\cdot, \zeta_{n_{0}}\right)
$$

and so this inequality must persist throughout $A \backslash\left\{\zeta_{n_{0}}\right\}$.

It follows that $\sum g_{A_{1}}\left(\zeta_{n}, \zeta_{n_{0}}\right)<M$ where this sum is extended over the same values of the index as the last one. But by the annular case we have considered, this means that $\left\{\zeta_{n}: \zeta_{n} \in A_{1}\right\}$ is an interpolation set for $A_{1}$.

The annuli $A_{2}, \cdots, A_{n}$ can be treated in a similar way, and the result follows from Theorem 5.6.

8. An embedding result. This final section is devoted to a careful delineation of the Riemann surfaces which admit an embedding of the particularly nice nature indicated in the introduction. We remark at the outset that it is clear we will be unable to obtain a delineation along purely topological lines. We have, for example, that the open unit disc and the complex plane are homeomorphic, but while the disc obviously admits an embedding of the desired kind, the plane does not. This last assertion follows from the fact that the plane carries no nonconstant bounded holomorphic functions whereas the surfaces which admit the embedding of the indicated kind have been seen to carry many such functions.

Our first result, which is purely topological in nature, is preliminary to our main result of this section, Theorem 8.2. If $S$ is a surface, we denote by $H_{1}(S, Z)$ its first singular homology group with coefficients in $Z$, the integers.

THEOREM 8.1. Let $S$ be an open Riemann surface such that $H_{1}(S, Z)$ is finitely generated. Then there exists a compact Riemann surface $R_{0}$ and an analytic homeomorphism $\phi$ of $S$ into $R_{0}$ such that $R_{0} \mid \phi[S]$ consists of finitely many closed discs which have analytic boundaries together with finitely many isolated points.

Proof. We begin by observing that there exists an analytic simple closed curve $\beta$ in $S$ with the property that $S \backslash \beta$ consists of two disjoint subsurfaces $S_{1}$ and $S_{2}$ of $S$ such that $S_{1} \cup \beta$ is a compact set and such that $S_{2}$ is homeomorphic to a region in the plane. 
The existence of the curve $\beta$ may be concluded as follows. Since $H_{1}(S, Z)$ is finitely generated, there is a compact surface $S_{0}$ and a not necessarily analytic homeomorphism $h$ of $S$ into such that $S_{0} \backslash h[S]$ consists of finitely many points $p_{1}, \cdots, p_{m}$. (For this point, see [2, p. 104].) We can pass an arc $\lambda$ through the points $p_{1}, \cdots, p_{m}$. If we then widen $\lambda$ into a thin open ribbon, say $A$, the set $A$ will be homeomorphic to a subset of the plane. Furthermore $S_{0} \backslash A$ is compact. We choose $A$ so that $\partial h^{-1}[A]$ is an analytic simple closed curve; this curve has the desired properties.

Since $S_{\mathbf{2}}$ is homeomorphic to a planar region, there is a conformal, one-to-one map $g$ of $S_{2}$ into the plane. (Cf. Theorem 9.1 of [16].) But now, by a classical theorem from the theory of conformal mapping [13, p. 377] there exists a conformal map $h$ of $g\left[S_{2}\right]$ onto a domain contained in the unit disc and bounded by circles, some of which may be degenerate. The map $h$ can be chosen so that if $f=h \circ g$, then $f$ is continued across $\beta$ and maps $\beta$ onto $\Lambda$. By identifying the point $z$ in $S_{2}$ with the point $f(z)$ in $U$, we sew $S$ and $U$ together analytically to obtain a compact Riemann surface $R_{0}$ which has the property of containing $S$ in the desired way. q.e.d.

We remark that this proof is an amalgamation of an earlier proof of the author's and a proof suggested by Professor Leo Sario (personal communication).

We can now formulate and prove the chief result of the present section. If $R$ is any Riemann surface, let $M(R)$ be the group of units of $H_{\infty}[R]$ so that

$$
M(R)=\left\{f \in H_{\infty}[R]: 1 / f \in H_{\infty}[R]\right\} .
$$

Let $E(R)$ be the subgroup of $M(R)$ consisting of all those $f$ such that for every integer $n$ other than 0 , there is $g \in H_{\infty}[R]$ such that $f=g^{n}$.

THEOREM 8.2. Let $S$ be Riemann surface such that $H_{1}(S, Z)$ is finitely generated and such that $H_{\infty}[S]$ does not reduce to the constants. Then there exists a compact Riemann surface $R_{0}$ and an analytic homeomorphism $\phi$ of $S$ into $R_{0}$ such that $R_{0} \mid \phi[S]$ consists of finitely many nondegenerate topological discs with analytic boundary if and only if $H_{1}(S, Z)$ and $M(S) / E(S)$ are isomorphic.

Proof. Before starting the proof, let us remark that it is the force of this theorem that the condition $H_{1}(S, Z) \simeq M(S) / E(S)$ is sufficient to guarantee that in the embedding given by Theorem 8.1, no isolated points occur in the complement. We also explicitly note that the conditions of the theorem are of a mixed algebraic and topological nature.

We begin by recalling that $H_{1}(S, Z)$ is a finitely generated, free abelian group, and so that $\operatorname{Hom}\left(H_{1}(S, Z), Z\right) \simeq H_{1}(S, Z)$.

We now establish the necessity of the condition. Suppose that $R_{0}$ is a compact surface and that $R$ is a region in $R_{0}$ such that $R_{0} \mid R=\Delta_{1} \cup \cdots \cup \Delta_{m}$ where each 
$\Delta_{j}$ is topologically a closed disc with analytic boundary. Let $p_{j} \in \Delta_{j}$ for each $j$, and suppose that $p_{j} \notin \vec{R}$. Let $\widetilde{R}=R_{0} \backslash\left\{p_{1}, \cdots, p_{m}\right\}$. Then $R$ and $\widetilde{R}$ are homeomorphic. Let $\tilde{\gamma}_{1}, \cdots, \tilde{\gamma}_{r}$ be a basis for $H_{1}(R, Z)$, and for each $j$, let $\gamma_{j}$ be a piecewise smooth cycle which represents $\tilde{\gamma}_{j}$. We may characterize the elements of $E(R)$ as those $f \in H_{\infty}[R]$ for which $\int_{\gamma_{j}} d f / f=0$ for each $j$.

We can define a homomorphism from $M(R)$ to $\operatorname{Hom}\left(H_{1}(R, Z), Z\right)$ as follows. If $f \in M(R)$, define $\phi_{f}$ by means of $\phi_{f}(\tilde{\gamma})=1 / 2 \pi i \int_{\gamma} d f / f$ where $\gamma$ is a piecewise smooth cycle in the homology class $\tilde{\gamma}$. The function $\phi_{f}$ is well defined, and it acts on $H_{1}(R, Z)$ as a $Z$-valued homomorphism. Furthermore, the map $f \rightarrow \phi_{f}$ is a homomorphism since if $f, g \in M(R)$, then $d(f g) / f g=d f / f+d g / g$. Note finally that the kernel of the homomorphism $f \rightarrow \phi_{f}$ from $M(R)$ to $\operatorname{Hom}\left(H_{1}(R, Z), Z\right)$ is exactly $E(R)$.

We say that the range of the homomorphism $f \rightarrow \phi_{f}$ is all of $\operatorname{Hom}\left(H_{1}(R, Z), Z\right)$. In order to show this, it would suffice to produce functions $F_{1}, \cdots, F_{m} \in M(R)$ such that $1 / 2 \pi i \int_{\gamma_{j}} d F_{k} / F_{k}=\delta_{j k}$. The existence of these $F_{j}$ is by no means a trivial point, but it can be concluded as follows: The cycles $\gamma_{1}, \cdots, \gamma_{m}$ constitute a basis not only for $H_{1}(R, Z)$, but also for $H_{1}(\tilde{R}, Z)$. By a theorem of Behnke and Stein $[14$, p. 198$]$, there exist analytic differentials $\omega_{j k}(j, k=1, \cdots, r)$ on $\widetilde{R}$ such that $\int_{\gamma_{j}} \omega_{j k}=2 \pi i \delta_{j k}$. Define $F_{j}$ on $\tilde{R}$ by $F_{j}(z)=\exp \int_{z_{0}}^{z} \omega_{j k}$ where $z_{0}$ is some fixed point of $\widetilde{R}$. Then $F$ is a well defined function on $\widetilde{R}$ and so may be regarded as an element of $H_{\infty}[R]$. But then we have

$$
\frac{1}{2 \pi i} \int_{\gamma_{j}} \frac{d F_{k}}{F_{k}}=\frac{1}{2 \pi i} \Delta_{\gamma_{j}} \log F_{k}=\delta_{j k}
$$

Thus we have that the map $f \rightarrow \phi_{f}$ is onto, and so

$$
M(R) / E(R) \simeq \operatorname{Hom}\left(H_{1}(R, Z), Z\right) \simeq H_{1}(R, Z) .
$$

We thus have the necessity of the condition.

To prove sufficiency, we shall make use of Theorem 8.1. Suppose then that $S$ carries nonconstant holomorphic functions, that $H_{1}(S, Z)$ is finitely generated, and that $H_{1}(S, Z) \simeq M(S) / E(S)$. By Theorem 8.1, there is an analytic homeomorphism $\phi$ of $S$ into a compact Riemann surface $R_{0}$ such that $R_{0} \mid \phi[S]$ consists of finitely many discs together with, possibly, finitely many points. Since $S$ carries nonconstant bounded holomorphic functions, $R_{0} \mid \phi[S]$ does not reduce to a discrete set of points. Suppose then that $R_{0} \mid \phi[S]=\Delta_{1} \cup \cdots \cup \Delta_{m} \cup\left\{p_{1}, \cdots, p_{k}\right\}$. We put $R=\phi[S] \cup\left\{p_{1}, \cdots, p_{k}\right\}$. By the argument of the first half of the proof and by the fact that each $p_{j}$ is a removable singularity for all $f \in H_{\infty}[\phi[S]]$, we have that $M(\phi[S]) / E(\phi[S]) \simeq H_{1}(R, Z)$. But by hypcthesis $M(S) / E(S) \simeq H_{1}(S, Z)$ whence $M(S) / E(S) \simeq M(\phi[S]) / E(\phi[S])$. Consequently, $H_{1}(S, Z) \simeq H_{1}(R, Z)$. This, however, implies that $\left\{p_{1}, \cdots, p_{k}\right\}=\varnothing$, and we have our theorem. 


\section{REFERENCES}

1. L. V. Ahlfors, Open Riemann surfaces and extermal problems on compact subregions, Comment. Math. Helv. 24 (1950), 100-134.

2. L. V. Ahlfors and L. Sario, Riemann surfaces, Princeton Univ. Press, Princeton, N. J., 1960.

3. N. L. Alling, A proof of the corona conjecture for firite open Riemann surfaces, Bull. Amer. Math. Soc. 70 (1964), 110-112.

4. R. Arens, The closed maximal ideals of algebras of functions holomorphic on a Riemann surface, Rend. Cir. Mat. Palermo 7 (1958), 245-260.

5. H. Behnke and F. Sommer, Theorie der analytischen Funktionen, Springer-Verlag, Berlin, 1955.

6. L. Carleson, An interpolation problem for bounded analytic functions, Amer. J. Math. 80 (1958), 921-930.

7. - Interpolation by bounded analytic functions and the corona problem, Ann. of Math. (2) 76 (1962), 547-560.

8. - Research on interpolation problems, Technical Report, Department of Mathematics, Uppsala University, Uppsala, 1962.

9. N. Dunford and J. Schwartz, Linear operators, Vol. I, General theory, Interscience, New York, 1958.

10. L. Gillman, and M. Jerison, Rings of continuous functions, Van Nostrand, Princeton. N. J., 1960.

11. M. Heins, Fundumental polygons of fuchsian and fuchsoid groups, Ann. Acad. Sci. Fenn Ser. A I No. 337 (1964).

12. K. Hoffman, Banach spaces of analytic functions, Prentice-Hall, Englewood Cliffs, N. J., 1962.

13. A. Hurwitz and R. Courant, Funktionentheorie, Springer-Verlag, Berlin, 1922.

14. A. Pfluger, Theorie der Riemannschen Flächen, Springer-Verlag, Berlin, 1957.

15. H. L. Royden, The boundary values of analytic and harmonic functions, Math. Z. 78 (1962), 1-24.

16. G. Springer, Introduction to Riemann surfaces, Addison-Wesley, Reading, Mass., 1957.

17. E. L. Stout, Two theorems concerning functions holomorphic on multiply connected domains, Bull. Amer. Math. Soc. 69 (1963), 527-530.

18. —- Some theorems on bounded holomorphic functions, Bull. Amer. Math. Soc. 70 (1964), 419-421.

19. M. Tsuji, Potential theory in modern function theory, Maruzen, Tokyo, 1959.

UNIVERSITY OF WISCONSIN,

Madison, Wisconsin

YALE UNIVERSITY,

New Haven, Connecticut 